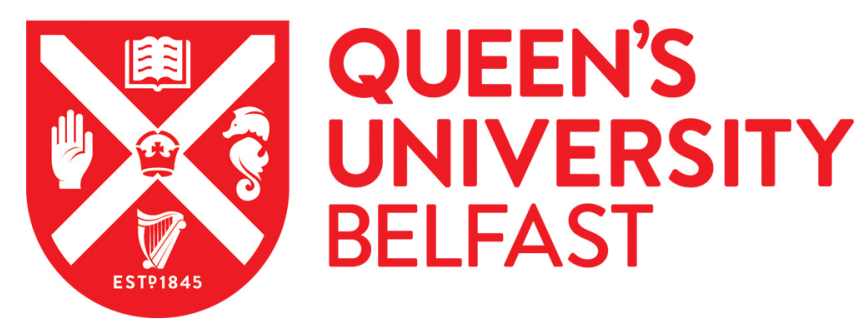

\title{
The BPtpA protein from Burkholderia cenocepacia belongs to a new subclass of low molecular weight protein tyrosine phosphatases
}

\begin{abstract}
Sanchez-Rodriguez, R., Gonzalez, G. M., Becerril-Garcia, M. A., Treviño-Rangel, R. D. J., Marcos-Vilches, A., Gonzalez-Pedrajo, B., Valvano, M. A., \& Andrade, A. (2020). The BPtpA protein from Burkholderia cenocepacia belongs to a new subclass of low molecular weight protein tyrosine phosphatases. Archives of Biochemistry and Biophysics. https://doi.org/10.1016/j.abb.2020.108277
\end{abstract}

Published in:

Archives of Biochemistry and Biophysics

Document Version:

Peer reviewed version

Queen's University Belfast - Research Portal:

Link to publication record in Queen's University Belfast Research Portal

Publisher rights

Copyright 2020 Elsevier

This manuscript is distributed under a Creative Commons Attribution-NonCommercial-NoDerivs License

(https://creativecommons.org/licenses/by-nc-nd/4.0/), which permits distribution and reproduction for non-commercial purposes, provided the author and source are cited.

\section{General rights}

Copyright for the publications made accessible via the Queen's University Belfast Research Portal is retained by the author(s) and / or other copyright owners and it is a condition of accessing these publications that users recognise and abide by the legal requirements associated with these rights.

Take down policy

The Research Portal is Queen's institutional repository that provides access to Queen's research output. Every effort has been made to ensure that content in the Research Portal does not infringe any person's rights, or applicable UK laws. If you discover content in the Research Portal that you believe breaches copyright or violates any law, please contact openaccess@qub.ac.uk. 


\title{
Manuscript Details
}

\section{Manuscript number}

Title
YABBI_2019_1012_R1

The BPtpA protein from Burkholderia cenocepacia belongs to a new subclass of low molecular weight protein tyrosine phosphatases

Short communication

\section{Article type}

\begin{abstract}
Low molecular weight protein tyrosine phosphatases (LMW-PTP) are ubiquitous enzymes found across a spectrum of genera from prokaryotes to higher eukaryotes. LMW-PTP belong to the Cys-based PTP class II protein family. Here, we show that LMW-PTP can be categorized into two different groups, referred as class II subdivision I (class II.I) and subdivision II (class II.II). Using to BPtpA from the opportunistic pathogen Burkholderia cenocepacia, as a representative member of the LMW-PTP class II.I, we demonstrated that four conserved residues (W47, H48, D80, and F81) are required for enzyme function. Guided by an in silico model of BPtpA, we show that the conserved residues at $\alpha 3$-helix (D80 and F81) contribute to protein stability, while the other conserved residues in the W-loop (W47 and H48) likely play a role in substrate recognition. Overall, our results provide new information on LMW-PTP protein family and establish B. cenocepacia as a suitable model to investigate how substrates are recognized and sorted by these proteins.
\end{abstract}

\section{Keywords}

Taxonomy

Corresponding Author

Order of Authors

Suggested reviewers
Burkholderia cenocepacia; tyrosine phosphorylation; tyrosine phosphatase.

Protein Classification, Biomolecules

Angel Andrade

Rebeca Sánchez-Rodríguez, Gloria González, Miguel Becerril-García, Rogelio Treviño-Rangel, Arely Marcos-Vilchis, Bertha González-Pedrajo, Miguel Valvano, Angel Andrade

Andrés Alonso, Andrew Fenton, Leonilde Moreira, Yossef Av-Gay

\section{Submission Files Included in this PDF}

\section{File Name [File Type]}

Rebuttal letter_YABBI_2019_1012.pdf [Response to Reviewers]

RV_Changes_Marked_YABBI_2019_1012.pdf [Revised Manuscript with Changes Marked]

RV_YABBI_2019_1012.docx [Manuscript File]

Author Statement.docx [Author Statement]

Supplemental Material ABB2019.docx [Supporting File]

To view all the submission files, including those not included in the PDF, click on the manuscript title on your EVISE Homepage, then click 'Download zip file'. 


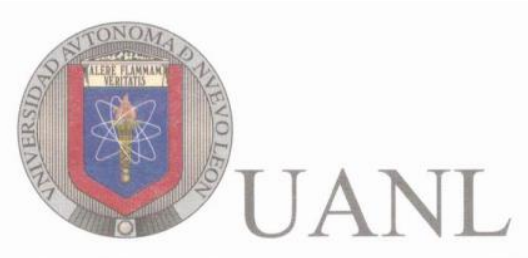

UNIVERSIDAD AUTÓNOMA DE NUEVO LEÓN
Angel Andrade

Associate Professor

Department of Microbiology,

School of Medicine, UANL

Phone: +52 (81) 83294166

Email: angel.andradet@uanl.edu.mx

15 January 2020

Dr. Paul Fitzpatrick, Editor in Chief

Archives of Biochemistry and Biophysics

Dear Dr. Fitzpatrick,

RE: YABBI_2019_1012

Thank you very much for your email of December 30, 2019. Please find enclosed our resubmission addressing the reviewers' comments (herein RV). Below are our point-by-point responses to the reviewers' comments.

\section{$\underline{\text { Reviewer \#1 }}$}

We thank this reviewer for his/her editorial suggestions, which we have implemented in the RV.

1. introduction section is longer than required.

This section and the References list have been shortened in the RV.

2. Discussion section is longer than required.

This section has been shortened in the RV.

3. In figures 2 and 3 it in western blots it should be indicated how many times they were run.

The information was included in Figure legends 2 and 3 of the RV.

\section{Reviewer \#2}

We thank this reviewer for stating our findings were straightforward and constitute a substantial contribution to the field.

\section{Critiques}

1. Figure 3A: The relative darkness of the bands in Figure $3 A$ is meaningless without context of how the gel was prepared. My guess is that a fixed volume of protein solution was loaded after a fixed volume of elution buffer was used to elute the different proteins. But this is not stated. The experimental details should be stated and the purified protein yield per liter of culture for each of the mutants should be specified.

Agreed. Experimental details regarding sample preparation for SDS-PAGE were included (see 2nd paragraph of page 7 in the RV). Also, the protein yield per liter of culture for each protein version was incorporated (see 2 nd paragraph of page 11 in the RV).

2. Figure 3C: It is not clear that the bands for the more highly expressing proteins in Figure 3C (WT and $D 127 A)$ are within the dynamic range of quantitation. For example, it does looks WT is somewhat 
depleted at 60 minutes compared to 5 minutes, but it is impossible to tell since the starting point (time 0 ) is so much darker than that of the D80A and F81A bands. The authors should adjust their exposure times (or the amount of protein loaded) so that the 0 points of all four proteins are normalized in intensity. Alternatively, they should provide evidence that the WT and D127A bands are clearly within the dynamic range of quantitation.

The amount of protein was normalized in all samples based on total protein quantification. Such discrepancies at time 0 (the beginning of the experiment) showing lower intensity with D80A and F81 A replacements were reproducible. We consider that this observation also reflects the stability issues identified in these two proteins. For clarity and to better illustrate the temporal differences of mutants D80A and F81A vs. WT or D127A, we calculated the relative intensities of the bands by densitomety (see 3rd paragraph of page 8, and 2nd paragraph of page 12 in the RV), and the ratios for the final/initial signal intensities were included into Fig3C in the RV.

In closing, we would like to thank both reviewers for their useful comments that have helped us to definitely improve our manuscript. We feel we have addressed all of their comments and believe the RV meets the standard for acceptance in Archives of Biochemistry and Biophysics.

Thank you very much again for editing this manuscript.

Sincerely yours

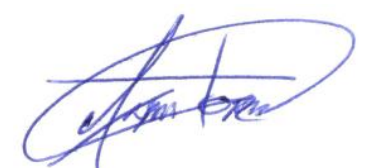

Angel Andrade, PhD

Associate Professor of Medical Microbiology 
The BPtpA protein from Burkholderia cenocepacia belongs to a new subclass of low molecular weight protein tyrosine phosphatases

Rebeca Sánchez-Rodríguez ${ }^{a}$, Gloria M. González ${ }^{a}$, Miguel A. Becerril-García ${ }^{a}$, Rogelio de J. Treviño-Rangel ${ }^{\mathrm{a}}$, Arely Marcos-Vilchis ${ }^{\mathrm{b}}$, Bertha González-Pedrajo ${ }^{\mathrm{b}}$, Miguel A. Valvano ${ }^{\mathrm{c}}$, and Angel Andrade $\mathrm{a}^{*}$

${ }^{a}$ Universidad Autónoma de Nuevo León, Facultad de Medicina, Departamento de Microbiología. Monterrey, Nuevo León, 64460, México.

${ }^{\text {b} D e p a r t a m e n t o ~ d e ~ G e n e ́ t i c a ~ M o l e c u l a r, ~ I n s t i t u t o ~ d e ~ F i s i o l o g i ́ a ~ C e l u l a r, ~ U n i v e r s i d a d ~ N a c i o n a l ~}$ Autónoma de México, Mexico City, 70-243, México.

${ }^{c}$ Wellcome-Wolfson Institute for Experimental Medicine, Queen's University Belfast, Belfast, BT9 7BL, United Kingdom.

\section{Corresponding author:}

Angel Andrade

Universidad Autónoma de Nuevo León, Facultad de Medicina, Departamento de Microbiología. Av. Francisco I. Madero s/n, Monterrey, Nuevo León, 64460, Mexico

Phone number: +52 (81) 83294177

E-mail address: angel.andradet@uanl.edu.mx 


\section{ABSTRACT}

Low molecular weight protein tyrosine phosphatases (LMW-PTP) are ubiquitous enzymes found across a spectrum of genera from prokaryotes to higher eukaryotes. LMW-PTP belong to the Cys-based PTP class II protein family. HereIn this work, we show that LMWPTP can be categorized into two different groups, referred as class II subdivision I (class II.I) and subdivision II (class II.II). Using to BPtpA from the opportunistic pathogen B:urkholderia cenocepacia, as a representative member of the LMW-PTP class II.I, we demonstrated that of four conserved residues (W47, H48, D80, and F81) are required for enzyme function. Guided by an in silico model of BPtpA, we show that the conserved residues at $\alpha 3$-helix (D80 and F81) contribute toare important for protein stability, while the other conserved residues in the W-loop (W47 and H48) likely play a role in substrate recognition. Overall, our results provide new informationextend the knowledge on LMWPTP protein family and establishpoints to B. cenocepacia as a suitable model to investigate evaluate how substrates are recognized and fsorted by these proteins.

\section{Highlights:}

Based on amino acid their-sequence similarity, we propose a new phylogenetic subclassification of the LMW-PTP into class II.I and class II.II.

Conserved W-loop residues are crucial for functionality of LMW-PTP class II.I from bacteria.

Conserved residues located at the $\alpha 3$-helix of the LMW-PTP class II.I contribute to maintain protein stability.

Keywords: Burkholderia cenocepacia; tyrosine phosphorylation; tyrosine phosphatase. 


\section{Introduction}

For over a decade, bacterial tyrosine phosphorylation has emerged as a key regulatory modification, contributing to signal integration, cellular response and adaptation [1]. The increasing number of bacterial phosphoproteomes have also uncovered a substantial abundance of prokaryotic tyrosine phosphorylated proteins $[2,3,4]$. To date, bacterial tyrosine phosphorylation has been mainly characterized in stress responses, cell division, DNA metabolism, and cell wall polysaccharide biosynthesis [2]. Prokaryotic proteins achieving tyrosine phosphorylation mainly belong to the bacterial tyrosine (BY) -kinase family [1]. BY-kinases enzymes-are widely distributed across multiple bacteria, in both Gram negative and Gram positive bacteria, and are-structurally and functionally distinct from their eukaryotic counterparts [3]. BY kinases become autophosphorylated on tyrosine residues located at the very end of its $C$ terminal, once phosphorylated they transfer the phosphate group to downstream targets [7].

Tyrosine phosphorylation is tightly controlled by the concerted action of protein-tyrosine phosphatases (PTP). In Proteobacteria, PTP mostly belong to the ubiquitous protein family termed low molecular weight (LMW)-PTP. The LMW-PTP are the only representatives of the Cys-based PTP class II, and are highly conserved across prokaryotes to higher eukaryotes [4]. In Firmicutes, the most prevalent PTP enzymes belong to the polymerase/histidinol phosphatase (PHP) protein family [5]. However, it is not uncommon that Gram-positive bacteria encode LMW-PTP, in addition to PHP enzymes [6].

LMW-PTP display a high degree of homology adopting a common fold, consisting of four stranded parallel $\beta$-sheets ( $\beta 1-4)$ connected by five $\alpha$-helices $(\alpha 1-5)$ [4]. All LMW-PTP contain a signature motif $\mathrm{CX}_{4} \mathrm{CR}$, which adopts a loop structure (P-loop), once the 
phosphate moiety of the substrate binds to the P loop, the first P-loop cysteine operates as a catalytic nucleophile, while. The active site motif is flanked by a second loop (D-loop), containing a conserved catalytic aspartate located on a second loop (D-loop), which facilitates departure of the phenolate moiety by acid catalysis [7]. A third loop (W-loop), forms a groove on the enzyme surface in the opposite wall of the active site cavity. Significantly, residues at the-W-loop residues arehave been recognized as determinants for substrate binding to specific ligands-in LMW-PTP from eukaryotes [8]. However, most of the prokaryotic LMW-PTP differ with their eukaryotic counterparts at the W-loop sequence [9]. Then, W-loop contribution to substrate recognition still needs to be clarified in bacteria.

The betaproteobacterium Burkholderia cenocepacia is an betaproteobacterium opportunistic pathogen belonging to a group of at least 22 genetically related species termed the Burkholderia cepacia complex (Bcc). Notably, Bcc bacteria cause severe lung infection in people with cystic fibrosis (CF) and other immunocompromised individuals [10]. B. cenocepacia chronic infections often result in a more rapid decline in lung function and eventually in a necrotizing pneumonia referred to as cepacia syndrome [15]. Successful treatment of B. cenocepacia infections is challenging due to the synergic contribution of intrinsic high levels of antibiotic resistance [11], combined with their extraordinary plasticity of $B$. cenocepacia to adapt and persist under hostile conditions in the airways and during chronic lung persistence-[12].

Tyrosine phosphorylation in B. cenocepacia remains poorly explored. We have described that B. cenocepacia K56-2 encodes two functional BY-kinases (BceF and BCAM1331), plus two LMW-PTP (BceD and BCAM0208), whose genes are distributed within the second chromoseme over three genetic clusters associated with exopolysaccharide (EPS) 
metabolism $[13,14]$. Further,Our previous work along with reports in other members of the Burkholderia genus, indicate that BeeF, BCAM1331, BceD, and BCAM0208 contribute to biofilm formation and EPS production [19,20,21]. Moreover, we have we described a third B. cenocepacia LMW-PTP, BCAL2200, which. It is encoded th the first chremeseme within a region presumably responsible for iron-sulfur [Fe-S] clusters assembly and without a close by encoded BY-kinase [13]. Notably, the genetic cluster including BCAL2200 is highly conserved in the Burkholderiaceae family, with no BY kinase encoded close to it. Specific roles were revealed fortø BCAL2200. Unlike BceD or BCAM0208 [14,15], BCAL2200 does not contribute to biofilm formation and is unable to achieve BceF dephosphorylation [14]. However, BCAL2200 was the only LMW-PTP required for growth under nutrient-limited conditions [14]. Based on the nomenclature used for homologous proteins and for simplicity, we propose to-renamed BCAL2200 as BPtpA ( Burkholderia phosphotyrosine phosphatase), this nomenclature will be used hereafter.

Thise present study focuses in understanding the molecular differences dictating BPtpA specificity. We revealed that LMW-PTP can be sub-categorized in two separated groups, referred to as class II.I and class II.II. -BPtpA belongs to the class II.I along with LMWPTP from eukaryotes and Firmicutes. Conserved amino acids were identified in LMW-PTP from class II.I and their contribution to BPtpA functionality was assessed.

\section{Material and methods}

\subsection{Bacterial strains, plasmids and growth conditions.}

Bacteria and plasmids used in this study are listed in Table S1. Bacteria were cultured in Luria broth (LB) or M9 minimal medium (Difco) supplemented with $0.4 \%$ glucose (Sigma), 
$2 \mathrm{mM} \mathrm{MgSO}_{4}$ (Sigma), and $100 \mu \mathrm{M} \mathrm{CaCl}_{2}$ (Sigma) at $37^{\circ} \mathrm{C}$ with shaking. If required, Escherichia coli cultures were supplemented with the following antibiotics (final concentrations): kanamycin $(40 \mu \mathrm{g} / \mathrm{ml})$ and tetracycline $(25 \mu \mathrm{g} / \mathrm{ml})$. B. cenocepacia cultures were supplemented with tetracycline $(100 \mu \mathrm{g} / \mathrm{ml})$. Growth in M9 minimal medium of $B$. cenocepacia $\triangle$ bptpA strain was evaluated as described [14] using the Epoch ${ }^{2}$ (BioTek) instrument.

\subsection{Construction of bptpA point mutants.}

Site direct mutagenesis was achieved by two steps PCR. Using pL2200 plasmid as template and the indicated primer pairs (Table S2). Amplicons from the first PCR round were purified and used as template for the second PCR with the primer pairs 6008 and 6009 (Table S2). High Fidelity polymerase (Jena, Bioscience) was used in all PCR reactions. Next, purified amplicons were double digested with $\mathrm{Nde} \mathrm{I}$ and $\mathrm{XbaI}$ (Invitrogen) and ligated into pDA17 plasmid using T4 DNA ligase (Invitrogen). Plasmids were mobilized into $B$. cenocepacia $\triangle$ bptpA strain by triparental mating using plasmid pRK2013 (Table S1). Cloning of bptpA point mutants in pET28 was achieved by PCR, using pDA17 based plasmids as template and primer pairs 7271 and 7272 (Table S1). Resulting amplicons were double digested with NcoI and XhoI (Invitrogen) and ligated into a similarly digested pET28 plasmid (Table S1). All DNA sequencing was completed at IPICYT, CONACYT (SLP, Mexico).

\subsection{Immunoblot analysis.}

Bacterial cultures grown during 15 hours in M9 minimal media were harvested by centrifugation. Resulting pellets were resuspended with PBS and total protein amount was determined using the Bio-Rad protein assay dye-binding reagent. Protein samples were 
adjusted (250 $\mu \mathrm{g}$ of total protein) and separated by $15 \%$ SDS-PAGE, transferred onto a polydivinylidine fluoride (PDVF) membrane (Merck), and probed with anti-FLAG M2 monoclonal antibody (Sigma) or with anti-enteropathogenic E. coli [16]. Secondary antibody was HRP-conjugated goat anti-mouse (Santa Cruz Biotechnology) or goat anti-rabbit (Sigma), respectively. Detection was performed using Immobilon Western chemiluminescent HRP substrate (Millipore), and bands were visualized on X-ray films (Kodak).

\subsection{Purification of recombinant proteins.}

Recombinant BPtpA enzyme or point mutant versions W47A, $\Delta$ W47, H48A, D80A, F81A, and D127A were purified as previously described [14] with minor modifications. Plasmids pAT44, pRS2, pRS3, pRS4, pRS5, pRS6, and pRS7 (Table S1) were expressed in E. coli BL21(DE3) (Table S1). Two hundred-milliliter cultures were grown in LB at $30^{\circ} \mathrm{C}$. Whereas for E. coli BL21(DE3) carrying pRS5 or pRS6 (expressing BPtpA D80A and F81A, respectively), the culture volume used was $500 \mathrm{ml}$. Once all cultures reached an $\mathrm{OD}_{600}$ of 0.7, $0.3 \mathrm{mM}$ IPTG (isopropyl- $\beta$-D-thiogalactopyranoside, Sigma) was added and growth was continued for $4 \mathrm{~h}$. Bacteria were harvested by centrifugation, and resulting pellets were resuspended in binding buffer $(\mathrm{BB} ; 20 \mathrm{mM}$ Tris- $\mathrm{HCl}, \mathrm{pH} 8.0,0.5 \mathrm{M} \mathrm{NaCl})$ containing a protease inhibitor cocktail (Jena, Bioscience) and lysed by sonication. Lysates were centrifuged for $60 \mathrm{~min}$ at $24,000 \times \mathrm{g}$ at $4^{\circ} \mathrm{C}$. The soluble fractions were applied to a Ni-NTA resin (Expedeon) for $30 \mathrm{~min}$ at $4^{\circ} \mathrm{C}$. After extensive washing with $\mathrm{BB}$ containing $40 \mathrm{mM}$

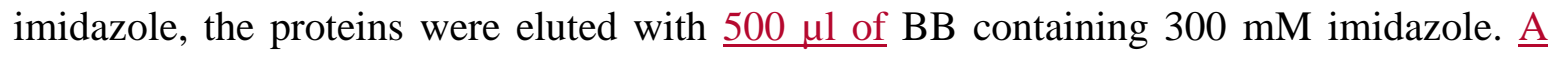
sample $(20 \mu \mathrm{l})$ of each eluted protein was mixed with 4 X SDS sample loading buffer $(6 \mu 1)$, separated by SDS-PAGE and stained by Coomassie brilliant blue. Purified recombinant 
proteins were dialyzed overnight at $4^{\circ} \mathrm{C}$ against 1.5 liter of TND buffer (50 mM Tris- $\mathrm{HCl}$, $\mathrm{pH}$ 8.0, $100 \mathrm{mM} \mathrm{NaCl}, 1 \mathrm{mM}$ dithiothreitol [DTT]). Protein concentration was determined by using the Bio-Rad protein assay dye-binding reagent.

\subsection{Phosphatase activity.}

Phosphatase activity was determined by monitoring, at $405 \mathrm{~nm}$, the amount of $p$-nitrophenol formed from $p$-nitrophenol phosphate as previously described [13]. Concentration of $p$ nitrophenol was estimated using a molar extinction coefficient of $1.78 \times 10^{4} \mathrm{M}^{-1} \mathrm{~cm}^{-1}$. Phosphatase activity was calculated from the slope obtained in time course analyses from three independent assays for each recombinant enzyme.

\subsection{Protein stability assay}

Fresh LB cultures of $B$. cenocepacia carrying plasmids pL2200, pbptpA $A_{\mathrm{D} 80 \mathrm{~A}}, \operatorname{pbptp} \mathrm{A}_{\mathrm{F} 81 \mathrm{~A}}$, or pbptpA $\mathrm{D}_{\mathrm{D} 127 \mathrm{~A}}\left(\right.$ Table $\mathrm{S} 1$ ), were grown at $37{ }^{\circ} \mathrm{C}$ until reaching an $\mathrm{OD}_{600}$ of 0.4 . One-ml culture was centrifuged as control. Then, protein synthesis was stopped by adding $12 \mu \mathrm{g} / \mathrm{ml}$ chloramphenicol (Sigma). Cultures were kept at $37^{\circ} \mathrm{C}$, and at different time intervals $1 \mathrm{ml}$ of culture was centrifuged and bacterial pellets were stored at $-20^{\circ} \mathrm{C}$. Protein samples were adjusted and analyzed by immunoblotting as described above. Western blot protein bands $\underline{\text { relative quantification was achieved using ImageJ software [17] and expressed as the ratios }}$ of the final/initial time interval signal intensities.

\subsection{Bioinformatics analysis and BPtpA structural modeling.}

Multiple protein sequence alignments were performed using MAFFT [18]. WebLogo 3.6 [19] was used to identify sequence conservation at specific positions and to compare residues between LMW-PTP classes. Bayesian inference of phylogeny was calculated using 
MRBAYES [20]. Structural model of BPtpA was built with the SWISS-MODEL server (http://swissmodel.expasy.org/) using VcLMWPTP-1 (45.6 \% identity with BPtpA) from Vibrio cholerae O395 (PDB accession number 4LRQ) as the template. Model refinement was performed using Chiron [21]. Model visualization, and figure preparation were performed with PyMol, Version 2.0 (Schrödinger, LLC).

\section{Results \\ 3.1 BPtpA belongs to the LMW-PTP class II.I.}

To clarify the functional differences between the three endogenous LMW-PTP of $B$. cenocepacia, BPtpA, BceD, and BCAM0208 [14], the amino acid sequences of these proteins were aligned with prototypical Cys-based PTP class II enzymes using Fast Fourier Transform. Based on the iterative sequence alignment, two clusters of LMW-PTP became evident (Fig. 1). The first clustergroup, consists mainly of LMW-PTP from eukaryotes and Firmicutes, while. In centrast, the second contains aremp is cempesed-only by prokaryotic enzymes (mostly from Gram-negative bacteria), including B. cenocepacia BceD and BCAM0208 (Fig. 1). Categorization of the LMW-PTP into two separated clusters was further supported by its evolutionary divergence, as revealed by Bayesian inference of phylogeny (Fig. S1).

Based on structural data, Lescop et al [9] have suggested a similar distinction; they classified eukaryotic and bacterial LMW-PTP as class I and class II, respectively. Considering that LMW-PTP belong to the Cys-based PTP class II, and in keeping with the Cys-based PTP class I categorization [22] we designated the two groups found in our analyses as class II subdivision I (class II.I) and subdivision II (class II.II). The class II.I 
groups proteins found in eukaryotes and the majority of the LMW-PTP from Gram-positive bacteria. However, a few Gram-negative bacterial proteins, including B. cenocepacia, BPtpA also belong to class II.I (Fig. 1 and Fig. S1). The LMW-PTP class II.I are distinguished primarily by (i) a conserved aromatic residue at the W-loop commonly followed by the positively charged residue histidine, plus (ii) a set of two consecutive aromatic residues at the D-loop (Fig. 1).

\subsection{Conserved amino acids in class II.I enzymes are important for BptpA functionality.}

To better understand the distinctive features of the two LMW-PTP groups, we examined the conserved residues within the class II.I not shared by class II.II proteins (Fig. 1). From this analysis, eight amino acid residues in BPtpA were selected for alanine replacement using site-directed mutagenesis: T44, W47, and H48 (at W-loop), D80 and F81 (at a3helix), F130 (at D-loop), and D141 and C147 (at $\alpha 5$-helix). Because an equivalent W-loop aromatic residue is absent in class II.II enzymes, we also constructed a version of BPtpA lacking W47 (Table S1).

We have previously shown that an enzymatically active C-terminal FLAG-tagged BPtpA version can restore the growth defect of B. cenocepacia $\Delta b p t p A$ strain under limiting nutrient conditions [14], indicating that the FLAG tag does not alter protein function. Therefore, plasmid pL2200 (Table S1), encoding the bptpAfLAG chimera was used as template for site-directed mutagenesis. Each plasmid derivative (Table S1) was mobilized into B. cenocepacia $\triangle b p t p A$ strain and their growth in M9 minimal medium was assessed (Fig. 2). The results showed that BPtpA mutant versions W47A, $\Delta$ W47, H48A, D80A, and F81A could not restore growth when expressed in B. cenocepacia $\Delta b p t p A$ strain, similar to BPtpA with the catalytic point mutation D127A, or the negative control plasmid pDA17 
(Fig. 2A). In contrast, alanine replacements in BptpA residues T44, F130, D141, or C147 did not alter protein functionality based on the ability of these constructs to restore the $\triangle b p t p A$ strain growth at comparable levels as the parental protein (Fig. 2B). Expression of all BPtpA replacement mutants was validated by immunoblotting using a monoclonal antibody against the FLAG-tag (Fig. 2C, upper panel). Detection of recombinant BPtpA versions was not uniform. Indeed, BPtpA with D80A and F81A replacements did not restore growth of $\triangle b p t p A$ strain, and also had reduced protein expression as compared to the wild-type enzyme (Fig. 2C, upper panel). This reduced signal was not due to differences in protein loading, as validated by detection of an unrelated cross-reacting protein by using a rabbit anti-E. coli serum (Fig. 2C, lower panel).

\subsection{Phosphatase activity and stability of BPtpA loss-of-function point mutants.}

BPtpA with substitutions W47A, $\Delta \mathrm{W} 47, \mathrm{H} 48 \mathrm{~A}, \mathrm{D} 80 \mathrm{~A}$, and F81A resemble the phenotype of the catalytic mutation D127A (Fig. 2A). Therefore, to rule out an enzymatic deficiency of this BPtpA protein versions, each corresponding gene was subcloned into an inducible vector (Table S1). Resulting recombinant proteins were purified by nickel affinity chromatography (Fig. 3A). Similar amount of recombinant protein was recovered from the different BPtpA versions, except for point mutants D80A and F81A. The purified protein yield (mg/l of culture) obtained for each version was as follows: wild-type $0.91 \pm 0.07$; $\underline{\text { W47A } 0.87 \pm 0.06 ; \Delta \mathrm{W} 471.03 \pm 0.21 ; \mathrm{H} 48 \mathrm{~A} 1.12 \pm 0.13 ; \mathrm{D} 80 \mathrm{~A} 0.39 \pm 0.05 ; \mathrm{F} 81 \mathrm{~A} 0.28}$ \pm 0.08 ; and D127A $1.30 \pm 0.31$, for which a low protein yield was obtained (Fig. $3 \mathrm{~A}$ ).

Phosphatase activity of purified BPtpA point mutants was assessed using $p$-nitrophenol phosphate (Fig. 3B). The specific activity of BPtpA versions W47A, $\Delta$ W47, and H48A showed no significant differences when compared to wild-type enzyme $(1.37 \pm 0.19 \mu$ moles 
$\mathrm{min}^{-1} \mathrm{mg}^{-1}$ ). In contrast, the enzymatic activity of BPtpA D80A and F81A was abrogated, similarly to the BPtpA inactive version, D127A (Fig. 3B).

Based on the results obtained for BPtpA versions D80A and F81A, we speculated that protein stability or misfolding might be responsible for the loss of function of these two mutant proteins. To confirm this, we compared the stability of BPtpA wild-type, and inactive point mutants D80A, F81A, and D127A after protein synthesis arrest (Fig. 3C). Time-course analysis tetection of BPtpA D80A and F81A demenstrated revealed a continuous decrease over the analyzed-evaluated time period. In contrast, BPtpA wild-type or inactive point mutant D127A showed a constant signal during the length of the experiment (Fig. 3C). The relative densitometric quantification of the final/initial time interval intensities also evidenced the temporal detriment of BPtpA D80A and F81A, compared to wild-type or D127A (Fig. 3C).

\section{Discussion}

Tyrosine phosphorylation is a widespread, reversible post-transtational modification influencing multiple cellular functions. LMW PTP are ubiquitously distributed enzymes achieving tyrosine dephosphorylation over a large range of protein substrates. LMW-PTP are ubiquitous enzymes belonging to the Cys-based PTP class II family. They share, sharing high amino acid sequence identity and-plus a common fold [4]. Based on our $\underline{\text { results the cumulative evidence and the data presented here, we propose to sub-divide }}$ eategorize the LMW-PTP enzymes into two separated groups, referred as class II.I and class II.II. 
U Using to-BPtpA from B. cenocepacia, as a representative member of the LMW-PTP class

II.I, wea mutational analysis of conserved amino acids was carried out. Accordingly, we demonstrated a key biological role of the BPtpA-residues W47, H48, D80, and F81., during $B$. cenocepacia $\Delta b p t p A$ strain growth under nutritional stress.

Our BPtpA structural model (Fig. 4A and B) suggests that W-loop residues W47 and H48, are part of an exposed wall regulating the selective access of phosphorylated substrates to the inward phosphatase active site. Indeed, the structural orientation and charge topology of the W-loop in eukaryotic LMW-PTPs were implicated in the has been related with recognition ofte specific ligands in different LMW PTP from eukaryotes-[8]. For instance, the corresponding residue to BPtpA H48 imparts charge to the active site and isoenzyme kinetic specificity in HPTP-A and HPTP-B human isoforms Particularly, it has been reported for human isoforms, HPTP $\triangle$ and HPTP B, that the corresponding residue to BPtpA H48, imparts charge distribution around the active site and isoenzyme kinetic specificity $[23,24]$. Further, P-loop ligand binding triggers a large reorientation of the residue W48 in MptpA from M. tuberculosis MptpA W48 residue-(which corresponds to W47 in BPtpA) and the D-loop, provoking the transition from an open state to a closed conformation that and therefore-modulatesing the surface of the substrate binding pocket $[25]$.

A triad of conserved aromatic residues at W- and D-loop is eonsidered the hallmark of LMW-PTP class II.I. Hence, a common mechanism involving these three residues during proper ligand positioning might be anticipated_in LMW-PTP class H.I. In this context Accordingly, strong $\pi-\pi$ stacking interactions implicating W- and D-loop aromatic residues of HPTP-A, were recently-identified as critical for binding the benzothiazole ring of 
different pTyr mimetic inhibitors [26]. The notion of a common substrate interaction in LMW PTP class II.I is further supported by the concerted movement of the corresponding aromatic residues of MptpA after ligand binding [30]. Nonetheless, alanine replacement of the second D-loop aromatic residue (F130) did not affect either BPtpA capability to restore $\triangle b p t p A$ strain growth defect (Fig. 2B) or phosphatase activity (data not shown), indicating that at least forin BPtpA, the first D-loop aromatic residue (Y129) must provide the main site for substrate binding. In agreement-with this notion, alanine substitution of the corresponding residue in Ltp1 from the yeastSaccharomyces cerevisiae, caused a marked drop in the $k_{\text {cat }} / K_{\mathrm{m}}$ value [27]. Moreover, the first D-loop aromatic residue is conserved in LMW-PTP class II.II and it has been implicated in substrate binding [28,29]. The D-loop aromatic residues could also play a role in protein dimerization, as suggested for the bovine LMW-PTP, BPTP [30], although an alternative mode of dimerization was reported for $V c$ LMWPTP-1 from $V$. cholerae [31]. Therefore, the contribution of D-loop residues to oligomerization still needs clarification.

Our data reveal a critical role of two BPtpA $\alpha 3$-helix conserved residues, D80 and F81. According to our BptpA model (Fig. 4A and C), both residues are located at the interior of the protein. In particular, the benzyl side chain of F81 is buried into a hydrophobic core encompassing hydrophobic residues V76 and L98 (Fig. 4C), which are highly conserved in all LMW-PTP. Moreover, the BPtpA model suggests that D80 forms a salt bridge with the charged residue R74 (Fig. 4C). An equivalent interaction is present in different LMW-PTP class II.I structures, including human, bovine, mouse, etc. (data not shown). Together, our observations the current evidence from our work-suggests that the $\alpha 3$-helix conserved residues are necessary for LMW-PTP class II.I protein stability. The uncovered functional 
relevance of the $\alpha 3$-helix region could provide an alternative option for rational drug design against LMW-PTP, especially considering the difficulties encountered for LMW-PTP selective drug targeting involving the charged nature and high degree of homology of PTP catalytic sites [32].

In summary, our findings indicate that BPtpA belongs to the eukaryotic like LMW PTP group, here termed as class II.I. BPtpA mutational analysis confirms the relevance of the W loop residues in bacterial class II.I phosphatases and revealed that conserved residues located at a 3 helix are critical for proper enzyme function.Efforts are under way to systematically identify protein substrates of B. cenocepacia phosphorylated proteins, including BPtpA substrates important for adaptation to nutrient starvation.

\section{Declaration of competing interest}

All authors declare that they have no conflicts of interest

\section{Acknowledgements}

We acknowledge M.S. Maria F. Flores, M.S. José F. Espinosa, and Dra. Norma Espinosa Sánchez for their expert technical assistance.

\section{Funding}

This study was supported by a grant from SEP-PRODEP [511-6/17-7538] to A.A.

\section{References}

[1] E. Bechet, S. Guiral, S. Torres, I. Mijakovic, A.J. Cozzone, C. Grangeasse, Tyrosinekinases in bacteria: from a matter of controversy to the status of key regulatory enzymes, Amino Acids 37 (2009) 499-507. 
[2]A.M.Hansen, R. Chaerkady, J. Sharma, J.J. Díaz Mejía, N. Tyagi, S. Renuse, H.K. Jacob, S.M. Pinte, N.A. Sahasrabuddhe, M.S. Kim, B. Delanghe, N. Srinivasan, A. Emili, J.B. Kaper, A. Pandey, The Escherichia coli phosphotyrosine proteome relates to core pathways and virulence, PLeS Patheg 9 (2013) e1003403.

[3] A.J. Standish, M.Y. Teh, E.N.H. Tran, M.T. Doyle, P.J. Baker, R. Morona, Unprecedented Abundance of Protein Tyrosine Phosphorylation Modulates Shigella flexneri Virulence, JMol Biol 428 (2016) 4197-4208.

41 A. Ravichandran, N. Sugiyama, M. Tomita, S. Swarup, Y. Ishihama, Ser/Thr/Tyr phosphoproteome analysis of pathogenic and non pathogenic Pseudomonas species, Proteomics 9 (2009) 2764-2775.

[2] I. Mijakovic, J. Deutscher, Protein-tyrosine phosphorylation in Bacillus subtilis: a 10year retrospective, Front Microbiol 6 (2015) 18.

[3] C. Grangeasse, S. Nessler, I. Mijakovic, Bacterial tyrosine kinases: evolution, biological function and structural insights, Philos Trans R Soc Lond B Biol Sci 367 (2012) 2640-2655. [7]P. Doublet, C. Grangeasse, B. Obadia, E. Vaganay, A.J. Cozzone, Structural organization of the protein-tyrosine autokinase Wze within Escherichia coli cells, J BiolChem 277 (2002) $\underline{3733937348 .}$

[4] A. Caselli, P. Paoli, A. Santi, C. Mugnaioni, A. Toti, G. Camici, P. Cirri, Low molecular weight protein tyrosine phosphatase: Multifaceted functions of an evolutionarily conserved enzyme, Biochim Biophys Acta 1864 (2016) 1339-1355.

[5] L. Aravind, E.V. Koonin, Phosphoesterase domains associated with DNA polymerases of diverse origins, Nucleic Acids Res 26 (1998) 3746-3752.

[6] A.J. Cozzone, C. Grangeasse, P. Doublet, B. Duclos, Protein phosphorylation on tyrosine in bacteria, Arch Microbiol 181 (2004) 171-181. 
[7] K. Kolmodin, J. Åqvist, The catalytic mechanism of protein tyrosine phosphatases revisited, FEBS Lett 498 (2001) 208-213.

[8] R. Maccari, R. Ottana, Low molecular weight phosphotyrosine protein phosphatases as emerging targets for the design of novel therapeutic agents, J Med Chem 55 2-22.

[9] E. Lescop, Y. Hu, H. Xu, W. Hu, J. Chen, B. Xia, C. Jin, The solution structure of Escherichia coli Wzb reveals a novel substrate recognition mechanism of prokaryotic low molecular weight protein-tyrosine phosphatases, J Biol Chem 281 (2006) 19570-19577.

[10] P. Drevinek, E. Mahenthiralingam, Burkholderia cenocepacia in cystic fibrosis: epidemiology and molecular mechanisms of virulence, Clin Microbiol Infect 16 (2010) 821830.

H15] J.M. Courtney, K.E. Dumbar, A. MeDowell, J.E. Moore, T.J. Warke, M. Stevenson, J.S. Elborn, Clinical outcome of Burkholderia cepacia complex infection in cystic fibrosis adults, JCyst Fibres 3 (2004) 93-98.

[11] V.C. Scoffone, L.R. Chiarelli, G. Trespidi, M. Mentasti, G. Riccardi, S. Buroni, Burkholderia cenocepacia Infections in Cystic Fibrosis Patients: Drug Resistance and Therapeutic Approaches, Front Microbiol 8 (2017) 1592.

[12] N.P. Mira, A. Madeira, A.S. Moreira, C.P. Coutinho, I. Sá-Correia, Genomic expression analysis reveals strategies of Burkholderia cenocepacia to adapt to cystic fibrosis patients' airways and antimicrobial therapy, PLoS One 6 (2011) e28831.

[13] A. Andrade, M.A. Valvano, A Burkholderia cenocepacia gene encoding a nonfunctional tyrosine phosphatase is required for the delayed maturation of the bacteriacontaining vacuoles in macrophages, Microbiology 160 (2014) 1332-1345.

[14] A. Andrade, F. Tavares-Carreon, M. Khodai-Kalaki, M.A. Valvano, Tyrosine Phosphorylation and Dephosphorylation in Burkholderia cenocepacia Affect Biofilm 
Formation, Growth under Nutritional Deprivation, and Pathogenicity, Appl Environ Microbiol 82 (2016) 843-856.

[15] A.S. Ferreira, J.H. Leitão, S.A. Sousa, A.M. Cosme, I. Sá-Correia, L.M. Moreira, Functional analysis of Burkholderia cepacia genes bceD and bceF, encoding a phosphotyrosine phosphatase and a tyrosine autokinase, respectively: role in exopolysaccharide biosynthesis and biofilm formation, Appl Environ Microbiol 73 (2007) 524-534.

211 M. Fazli, Y. McCarthy, M. Givskov, R.P. Ryan, T. Tolker Nielsen, The exopolysaccharide gene cluster Bcam1330-Bcam1341 is involved in Burkholderia cenocepacia biofilm formation, and its expression is regulated by c di-GMP and Bcam1349, Mierebiologyopen 2(2013) 105122.

[16] M. Romo-Castillo, A. Andrade, N. Espinosa, J. Monjarás Feria, E. Soto, M. DíazGuerrero, B. Gonzaléz-Pedrajo, EscO, a Functional and Structural Analog of the Flagellar FliJ Protein, Is a Positive Regulator of EscN ATPase Activity of the Enteropathogenic Escherichia coli Injectisome, J Bacteriol 196 (2014) 2227-2241.

[17] C.A. Schneider, W.S. Rasband, K.W. Eliceiri, NIH Image to ImageJ: 25 years of image analysis, Nat Methods 9 (2012) 671-675.

[18] K. Katoh, J. Rozewicki, K.D. Yamada, MAFFT online service: multiple sequence alignment, interactive sequence choice and visualization, Brief Bioinform (2017).

[19] G.E. Crooks, G. Hon, J.M. Chandonia, S.E. Brenner, WebLogo: a sequence logo generator, Genome Res 14 (2004) 1188-1190.

[20] J.P. Huelsenbeck, F. Ronquist, MRBAYES: Bayesian inference of phylogenetic trees, Bioinformatics 17 (2001) 754-755. 
[21] S. Ramachandran, P. Kota, F. Ding, N.V. Dokholyan, Automated minimization of steric clashes in protein structures, Proteins 79 (2011) 261-270.

[22] A. Alonso, R. Pulido, The extended human PTPome: a growing tyrosine phosphatase family, FEBS J 283 (2016) 1404-1429.

[23] P. Cirri, T. Fiaschi, P. Chiarugi, G. Camici, G. Manao, G. Raugei, G. Ramponi, The molecular basis of the differing kinetic behavior of the two low molecular mass phosphotyrosine protein phosphatase isoforms, J Biol Chem 271 (1996) 2604-2607.

[24] A.P. Zabell, A.D. Schroff, Jr., B.E. Bain, R.L. Van Etten, O. Wiest, C.V. Stauffacher, Crystal structure of the human B-form low molecular weight phosphotyrosyl phosphatase at 1.6-Å resolution, J Biol Chem 281 (2006) 6520-6527.

[25] T. Stehle, S. Sreeramulu, F. Löhr, C. Richter, K. Saxena, H.R. Jonker, H. Schwalbe, The apo-structure of the low molecular weight protein-tyrosine phosphatase A (MptpA) from Mycobacterium tuberculosis allows for better target-specific drug development, J Biol Chem 287 (2012) 34569-34582.

[26] R. He, J. Wang, Z.H. Yu, R.Y. Zhang, S. Liu, L. Wu, Z.Y. Zhang, Inhibition of Low Molecular Weight Protein Tyrosine Phosphatase by an Induced-Fit Mechanism, J Med Chem 59 (2016) 9094-9106.

[27] P. Paoli, A. Modesti, F. Magherini, T. Gamberi, A. Caselli, G. Manao, G. Raugei, G. Camici, G. Ramponi, Site-directed mutagenesis of two aromatic residues lining the active site pocket of the yeast Ltp1, Biochim Biophys Acta 1770 (2007) 753-762.

[28] G. Hagelueken, H. Huang, I.L. Mainprize, C. Whitfield, J.H. Naismith, Crystal structures of Wzb of Escherichia coli and CpsB of Streptococcus pneumoniae, representatives of two families of tyrosine phosphatases that regulate capsule assembly, $\mathrm{J}$ Mol Biol 392 (2009) 678-688. 
[29] D.B. Temel, K. Dutta, S. Alphonse, J. Nourikyan, C. Grangeasse, R. Ghose, Regulatory interactions between a bacterial tyrosine kinase and its cognate phosphatase, $\mathrm{J}$ Biol Chem 288 (2013) 15212-15228

[30] L. Tabernero, B.N. Evans, P.A. Tishmack, R.L. Van Etten, C.V. Stauffacher, The structure of the bovine protein tyrosine phosphatase dimer reveals a potential self-regulation mechanism, Biochemistry 38 (1999) 11651-11658.

[31] S. Nath, R. Banerjee, U. Sen, Atomic resolution crystal structure of VcLMWPTP-1 from Vibrio cholerae O395: insights into a novel mode of dimerization in the low molecular weight protein tyrosine phosphatase family, Biochem Biophys Res Commun 450 (2014) 390395.

[32] Z.Y. Zhang, Drugging the Undruggable: Therapeutic Potential of Targeting Protein Tyrosine Phosphatases, Acc Chem Res 50 (2017) 122-129.

Figure 1. ( 2 column fitting image) 
A

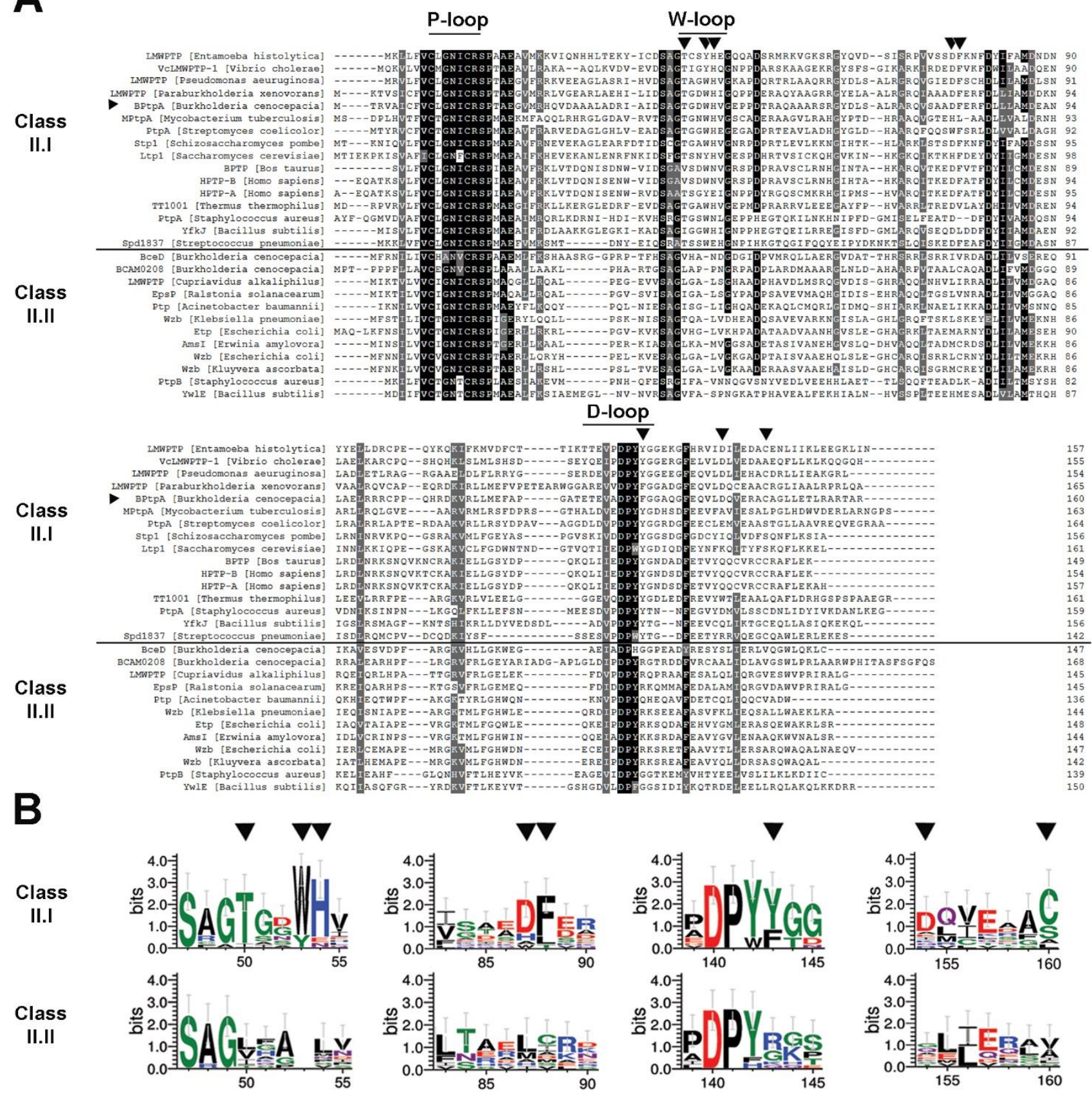

Figure 1. LMW-PTP enzymes can be categorized in two groups. Multiple sequence alignment of representative LMW-PTP enzymes. Straight horizontal line separates enzymes from Class II.I (above) and Class II.II (below). Residues composing the P-, W-, and Dloops are indicated. (B) Sequence logos for the corresponding regions in LMW-PTP Class II.I and Class II.II. Arrowheads denote BPtpA sequence, as well as the corresponding residues mutagenized in this study. 
Figure 2. (1 column fitting image)

A

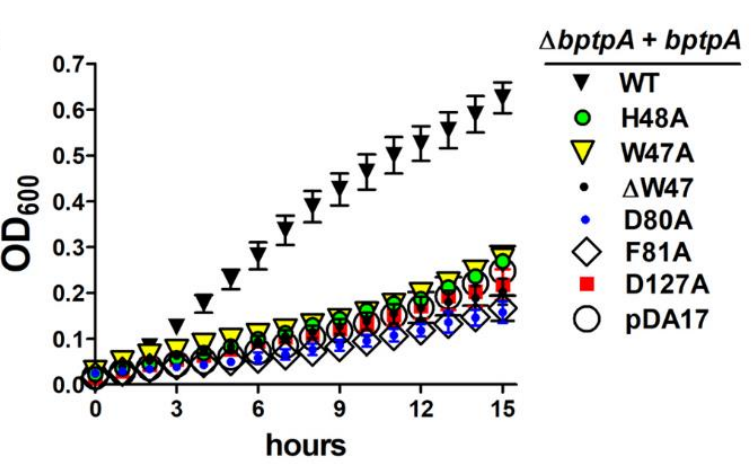

B

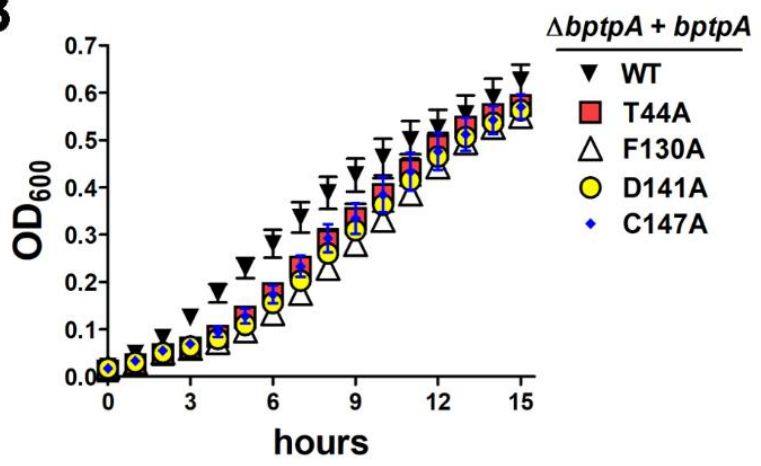

C
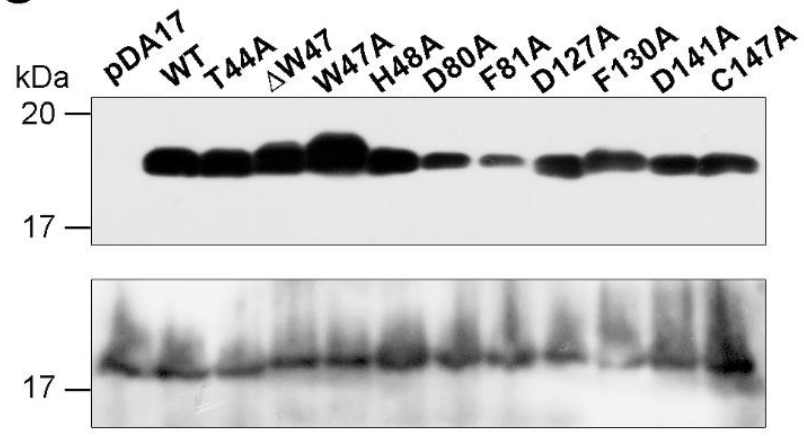

Figure 2. Conserved residues from LMW-PTP class II.I are required for BPtpA functionality. (A and B) Complementation of growth in M9 minimal medium of $B$. cenocepacia $\triangle b p t p A$ carrying pDA17-based plasmids. Data represent the mean \pm SEM from three independent experiments. (C) Immunoblot of total cell lysates of B. cenocepacia $\triangle b p t p A$ carrying pDA17-based plasmids growing in M9 minimal medium. Upper panel-and lower panels, immunoblot with anti-FLAG antibody or anti-E. coli serum, respectively. The positions of molecular mass markers are indicated on the left. Immunoblots were repeated at least three times. 
Figure 3. (1.5 column fitting image)
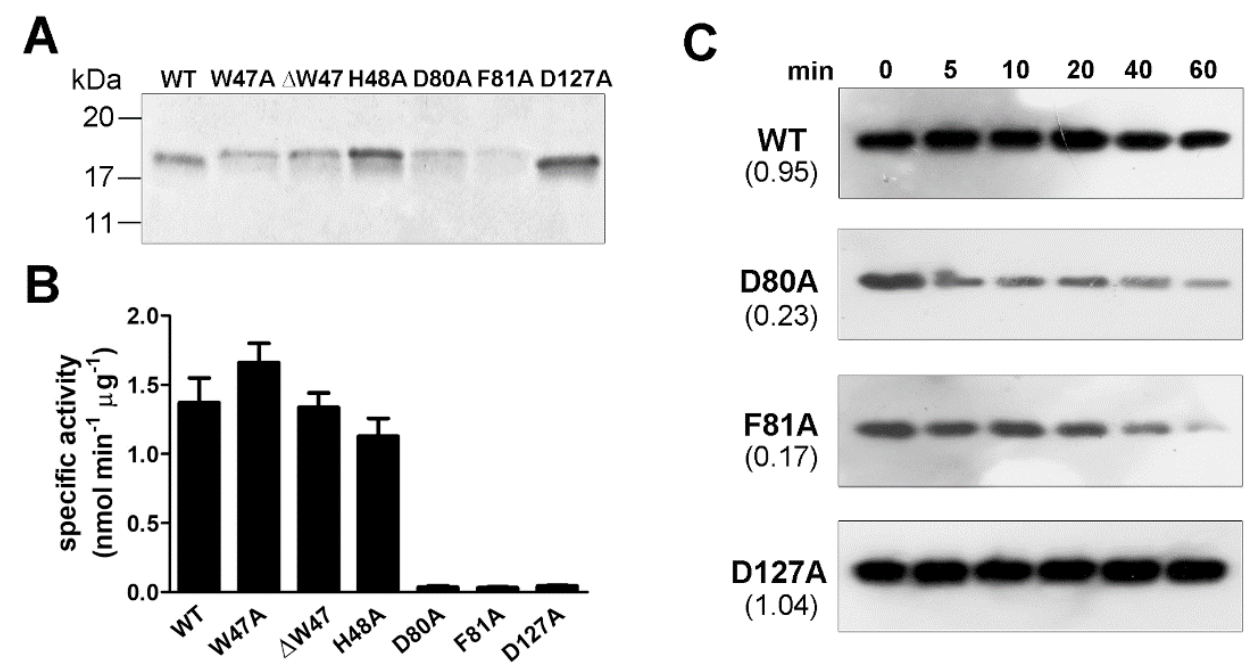

$(0.23)$

F81A

$(0.17)$

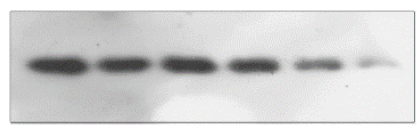

D127A

(1.04)

Figure 3. Phosphatase activity and stability of loss-of-function BPtpA point mutants. (A) Purification of recombinant LMW-PTP, each line corresponds to the indicated BPtpA version. The positions of molecular mass markers are indicated on the left. (B) In vitro phosphatase activity of purified BPtpA recombinant protein using $p$-nitrophenol phosphate (PNPP) as substrate. Data represent the mean \pm SEM from three independent experiments. (C) In vivo protein stability of BPtpA wild-type (WT) and inactive point mutant versions after stopping protein synthesis. At the indicated time intervals (minutes), a sample was collected and frozen. Immunoblot was performed with anti-FLAG antibody. The ratio final/initial signal intensity is indicated in parenthesis under the corresponding BPtpA version. Protein stability assays were performed three times. 
A

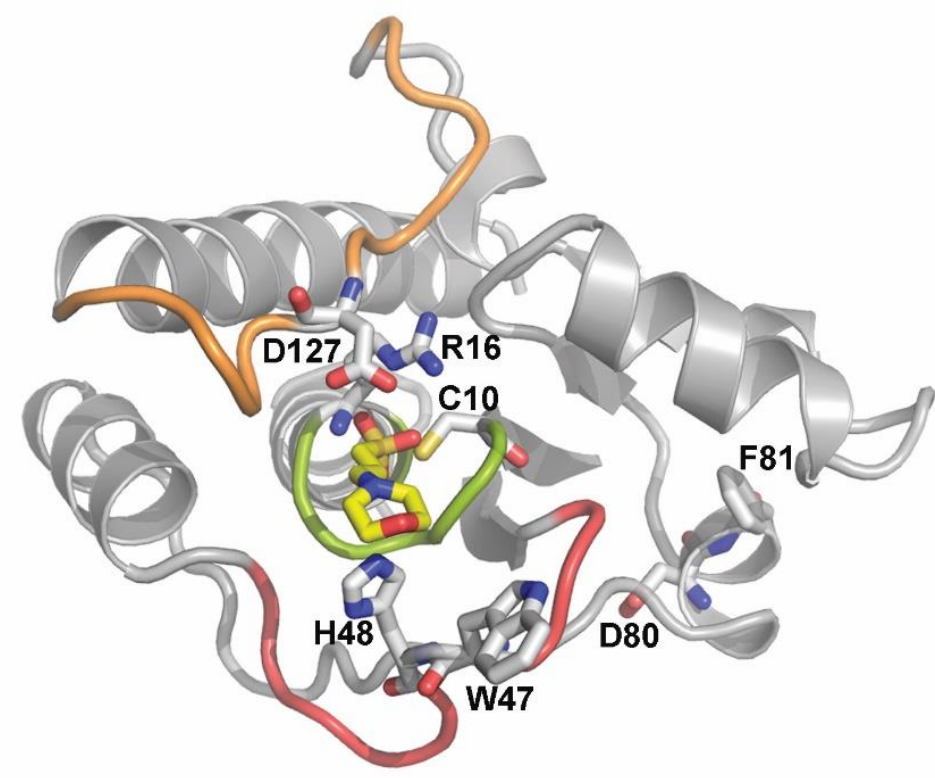

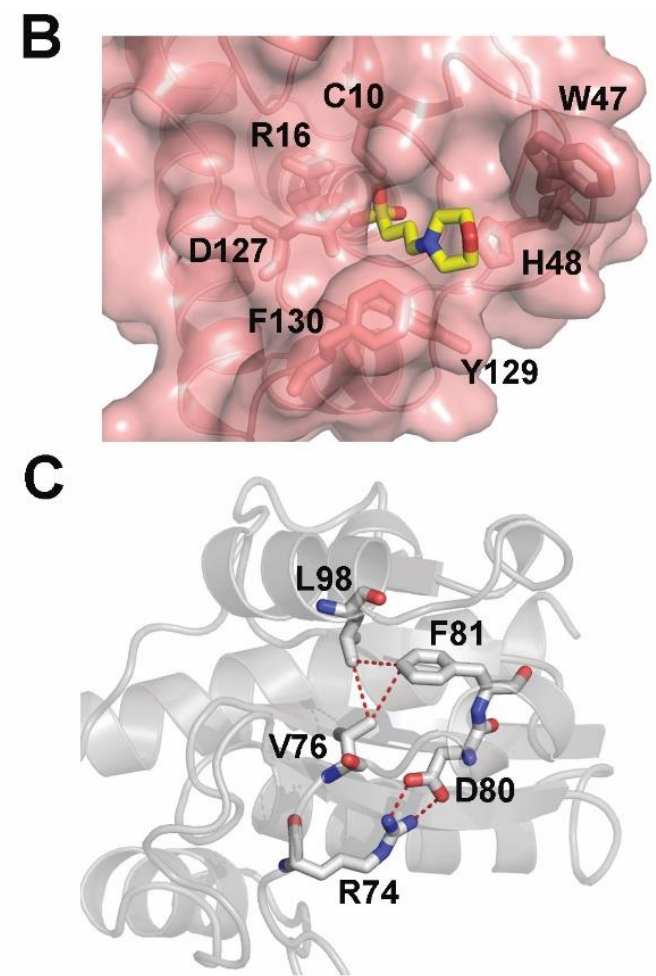

Figure 4. Mapping of functional residues on the BPtpA structural model. Modelled BPtpA is represented as a cartoon. (A) Functional residues identified in this work W47, H48, D80, and F81 are depicted as sticks. The P-, D-, and W-loop are colored in green, orange, and red, respectively. (B) Surface representation of the active-site cavity. Aromatic residues at W-loop, W47 and H48, and D-loop, Y129 and F130, are depicted as sticks. In (A and B) MOPS as ligand mimicking the substrate phosphotyrosine at the active site is shown as yellow sticks. Catalytic residues C10, R16 and D127 are depicted as sticks. (C) Close-up view of the $\alpha 3$-helix residues D80 and F81 residues and the presumptive interactions with R74, V76, and L98, respectively. 
The BPtpA protein from Burkholderia cenocepacia belongs to a new subclass of low molecular weight protein tyrosine phosphatases

Rebeca Sánchez-Rodríguez ${ }^{a}$, Gloria M. González ${ }^{a}$, Miguel A. Becerril-García $^{a}$, Rogelio de J. Treviño-Rangel ${ }^{\mathrm{a}}$, Arely Marcos-Vilchis ${ }^{\mathrm{b}}$, Bertha González-Pedrajo ${ }^{\mathrm{b}}$, Miguel A. Valvano ${ }^{\mathrm{c}}$, and Angel Andrade $\mathrm{a}^{*}$

aUniversidad Autónoma de Nuevo León, Facultad de Medicina, Departamento de Microbiología. Monterrey, Nuevo León, 64460, México.

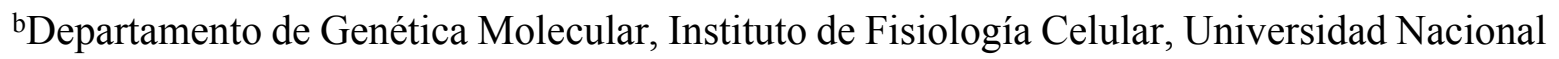
Autónoma de México, Mexico City, 70-243, México.

'Wellcome-Wolfson Institute for Experimental Medicine, Queen's University Belfast, Belfast, BT9 7BL, United Kingdom.

\title{
Corresponding author:
}

\author{
Angel Andrade \\ Universidad Autónoma de Nuevo León, Facultad de Medicina, Departamento de \\ Microbiología. Av. Francisco I. Madero s/n, Monterrey, Nuevo León, 64460, Mexico \\ Phone number: +52 (81) 83294177 \\ E-mail address: angel.andradet@uanl.edu.mx
}




\begin{abstract}
Low molecular weight protein tyrosine phosphatases (LMW-PTP) are ubiquitous enzymes found across a spectrum of genera from prokaryotes to higher eukaryotes. LMW-PTP belong to the Cys-based PTP class II protein family. Here, we show that LMW-PTP can be categorized into two different groups, referred as class II subdivision I (class II.I) and subdivision II (class II.II). Using to BPtpA from the opportunistic pathogen Burkholderia cenocepacia, as a representative member of the LMW-PTP class II.I, we demonstrated that four conserved residues (W47, H48, D80, and F81) are required for enzyme function. Guided by an in silico model of BPtpA, we show that the conserved residues at $\alpha 3$-helix (D80 and F81) contribute to protein stability, while the other conserved residues in the Wloop (W47 and H48) likely play a role in substrate recognition. Overall, our results provide new information on LMW-PTP protein family and establish B. cenocepacia as a suitable model to investigate how substrates are recognized and sorted by these proteins.
\end{abstract}

\title{
Highlights:
}

Based on amino acid sequence similarity, we propose a new phylogenetic sub-classification of the LMW-PTP into class II.I and class II.II.

Conserved W-loop residues are crucial for functionality of LMW-PTP class II.I from bacteria.

Conserved residues located at the $\alpha 3$-helix of the LMW-PTP class II.I contribute to maintain protein stability.

Keywords: Burkholderia cenocepacia; tyrosine phosphorylation; tyrosine phosphatase. 


\section{Introduction}

For over a decade, bacterial tyrosine phosphorylation has emerged as a key regulatory modification, contributing to signal integration, cellular response and adaptation [1]. To date, bacterial tyrosine phosphorylation has been mainly characterized in stress responses, cell division, DNA metabolism, and cell wall polysaccharide biosynthesis [2]. Prokaryotic proteins achieving tyrosine phosphorylation mainly belong to the bacterial tyrosine (BY) kinase family [1]. BY-kinases are widely distributed across multiple bacteria, and structurally and functionally distinct from their eukaryotic counterparts [3].

Tyrosine phosphorylation is tightly controlled by the concerted action of protein-tyrosine phosphatases (PTP). In Proteobacteria, PTP mostly belong to the ubiquitous protein family termed low molecular weight (LMW)-PTP. The LMW-PTP are the only representatives of the Cys-based PTP class II, and are highly conserved across prokaryotes to higher eukaryotes [4]. In Firmicutes, the most prevalent PTP enzymes belong to the polymerase/histidinol phosphatase (PHP) protein family [5]. However, it is not uncommon that Gram-positive bacteria encode LMW-PTP, in addition to PHP enzymes [6].

LMW-PTP display a high degree of homology adopting a common fold, consisting of four stranded parallel $\beta$-sheets $(\beta 1-4)$ connected by five $\alpha$-helices $(\alpha 1-5)$ [4]. All LMW-PTP contain a signature motif $\mathrm{CX}_{4} \mathrm{CR}$, which adopts a loop structure (P-loop). The first P-loop cysteine operates as a catalytic nucleophile, while a conserved catalytic aspartate located on a second loop (D-loop) facilitates departure of the phenolate moiety by acid catalysis [7]. A third loop (W-loop), forms a groove on the enzyme surface in the opposite wall of the active site cavity. W-loop residues are recognized as determinants for substrate binding in LMW-PTP from eukaryotes [8]. However, most of the prokaryotic LMW-PTP differ with 
their eukaryotic counterparts at the W-loop sequence [9]. Then, W-loop contribution to substrate recognition still needs to be clarified in bacteria.

The betaproteobacterium Burkholderia cenocepacia is an opportunistic pathogen belonging to a group of at least 22 genetically related species termed the Burkholderia cepacia complex (Bcc). Notably, Bcc bacteria cause severe lung infection in people with cystic fibrosis (CF) and other immunocompromised individuals [10] . Successful treatment of $B$. cenocepacia infections is challenging due to the synergic contribution of intrinsic high levels of antibiotic resistance [11], combined with their extraordinary plasticity to adapt and persist under hostile conditions in the airways [12].

Tyrosine phosphorylation in B. cenocepacia remains poorly explored. We have described that B. cenocepacia K56-2 encodes two functional BY-kinases (BceF and BCAM1331), plus two LMW-PTP (BceD and BCAM0208), whose genes are distributed over three genetic clusters associated with exopolysaccharide (EPS) metabolism $[13,14]$. Further, we described a third B. cenocepacia LMW-PTP, BCAL2200, which is encoded within a region presumably responsible for iron-sulfur [Fe-S] clusters assembly and without a close by encoded BY-kinase [13]. Specific roles were revealed for BCAL2200. Unlike BceD or BCAM0208 [14,15], BCAL2200 does not contribute to biofilm formation and is unable to achieve BceF dephosphorylation [14]. However, BCAL2200 was the only LMW-PTP required for growth under nutrient-limited conditions [14]. Based on the nomenclature used for homologous proteins and for simplicity, we renamed BCAL2200 as BPtpA ( Burkholderia phosphotyrosine phosphatase).

This study focuses in understanding the molecular differences dictating BPtpA specificity. We revealed that LMW-PTP can be sub-categorized in two separated groups, referred to as 
class II.I and class II.II. BPtpA belongs to the class II.I along with LMW-PTP from eukaryotes and Firmicutes. Conserved amino acids were identified in LMW-PTP from class II.I and their contribution to BPtpA functionality was assessed.

\section{Material and methods}

\subsection{Bacterial strains, plasmids and growth conditions.}

Bacteria and plasmids used in this study are listed in Table S1. Bacteria were cultured in Luria broth (LB) or M9 minimal medium (Difco) supplemented with 0.4\% glucose (Sigma), $2 \mathrm{mM} \mathrm{MgSO}_{4}$ (Sigma), and $100 \mu \mathrm{M} \mathrm{CaCl}_{2}$ (Sigma) at $37^{\circ} \mathrm{C}$ with shaking. If required, Escherichia coli cultures were supplemented with the following antibiotics (final concentrations): kanamycin $(40 \mu \mathrm{g} / \mathrm{ml})$ and tetracycline $(25 \mu \mathrm{g} / \mathrm{ml})$. B. cenocepacia cultures were supplemented with tetracycline $(100 \mu \mathrm{g} / \mathrm{ml})$. Growth in M9 minimal medium of $B$. cenocepacia $\triangle b p t p A$ strain was evaluated as described [14] using the Epoch ${ }^{2}$ (BioTek) instrument.

\subsection{Construction of bptpA point mutants.}

Site direct mutagenesis was achieved by two steps PCR. Using pL2200 plasmid as template and the indicated primer pairs (Table S2). Amplicons from the first PCR round were purified and used as template for the second PCR with the primer pairs 6008 and 6009 (Table S2). High Fidelity polymerase (Jena, Bioscience) was used in all PCR reactions. Next, purified amplicons were double digested with $\mathrm{NdeI}$ and $\mathrm{XbaI}$ (Invitrogen) and ligated into pDA17 plasmid using T4 DNA ligase (Invitrogen). Plasmids were mobilized into B. cenocepacia $\triangle b p t p A$ strain by triparental mating using plasmid pRK2013 (Table S1). Cloning of bptpA point mutants in pET28 was achieved by PCR, using pDA17 based plasmids as template and 
primer pairs 7271 and 7272 (Table S1). Resulting amplicons were double digested with NcoI and XhoI (Invitrogen) and ligated into a similarly digested pET28 plasmid (Table S1). All DNA sequencing was completed at IPICYT, CONACYT (SLP, Mexico).

\subsection{Immunoblot analysis.}

Bacterial cultures grown during 15 hours in M9 minimal media were harvested by centrifugation. Resulting pellets were resuspended with PBS and total protein amount was determined using the Bio-Rad protein assay dye-binding reagent. Protein samples were adjusted (250 $\mu \mathrm{g}$ of total protein) and separated by $15 \%$ SDS-PAGE, transferred onto a polydivinylidine fluoride (PDVF) membrane (Merck), and probed with anti-FLAG M2 monoclonal antibody (Sigma) or with anti-enteropathogenic E. coli [16]. Secondary antibody was HRP-conjugated goat anti-mouse (Santa Cruz Biotechnology) or goat anti-rabbit (Sigma), respectively. Detection was performed using Immobilon Western chemiluminescent HRP substrate (Millipore), and bands were visualized on X-ray films (Kodak).

\subsection{Purification of recombinant proteins.}

Recombinant BPtpA enzyme or point mutant versions W47A, $\Delta$ W47, H48A, D80A, F81A, and D127A were purified as previously described [14] with minor modifications. Plasmids pAT44, pRS2, pRS3, pRS4, pRS5, pRS6, and pRS7 (Table S1) were expressed in E. coli BL21(DE3) (Table S1). Two hundred-milliliter cultures were grown in LB at $30^{\circ} \mathrm{C}$. Whereas for E. coli BL21(DE3) carrying pRS5 or pRS6 (expressing BPtpA D80A and F81A, respectively), the culture volume used was $500 \mathrm{ml}$. Once all cultures reached an $\mathrm{OD}_{600}$ of 0.7, 0.3 mM IPTG (isopropyl- $\beta$-D-thiogalactopyranoside, Sigma) was added and growth was 
continued for $4 \mathrm{~h}$. Bacteria were harvested by centrifugation, and resulting pellets were resuspended in binding buffer $(\mathrm{BB} ; 20 \mathrm{mM}$ Tris- $\mathrm{HCl}, \mathrm{pH} 8.0,0.5 \mathrm{M} \mathrm{NaCl})$ containing a protease inhibitor cocktail (Jena, Bioscience) and lysed by sonication. Lysates were centrifuged for $60 \mathrm{~min}$ at $24,000 \times \mathrm{g}$ at $4^{\circ} \mathrm{C}$. The soluble fractions were applied to a Ni-NTA resin (Expedeon) for $30 \mathrm{~min}$ at $4^{\circ} \mathrm{C}$. After extensive washing with $\mathrm{BB}$ containing $40 \mathrm{mM}$ imidazole, the proteins were eluted with $500 \mu \mathrm{l}$ of BB containing $300 \mathrm{mM}$ imidazole. A sample $(20 \mu \mathrm{l})$ of each eluted protein was mixed with 4X SDS sample loading buffer $(6 \mu \mathrm{l})$, separated by SDS-PAGE and stained by Coomassie brilliant blue. Purified recombinant proteins were dialyzed overnight at $4^{\circ} \mathrm{C}$ against 1.5 liter of TND buffer $(50 \mathrm{mM}$ Tris- $\mathrm{HCl}$, pH 8.0, $100 \mathrm{mM} \mathrm{NaCl}, 1 \mathrm{mM}$ dithiothreitol [DTT]). Protein concentration was determined by using the Bio-Rad protein assay dye-binding reagent.

\subsection{Phosphatase activity.}

Phosphatase activity was determined by monitoring, at $405 \mathrm{~nm}$, the amount of $p$-nitrophenol formed from $p$-nitrophenol phosphate as previously described [13]. Concentration of $p$ nitrophenol was estimated using a molar extinction coefficient of $1.78 \times 10^{4} \mathrm{M}^{-1} \mathrm{~cm}^{-1}$. Phosphatase activity was calculated from the slope obtained in time course analyses from three independent assays for each recombinant enzyme.

\subsection{Protein stability assay}

Fresh LB cultures of B. cenocepacia carrying plasmids pL2200, pbptp $A_{\mathrm{D} 80 \mathrm{~A}}, \mathrm{pbptp}_{\mathrm{F} 81 \mathrm{~A}}$, or pbptpA $\mathrm{D}_{\mathrm{D} 127 \mathrm{~A}}$ (Table $\mathrm{S} 1$ ), were grown at $37^{\circ} \mathrm{C}$ until reaching an $\mathrm{OD}_{600}$ of 0.4 . One-ml culture was centrifuged as control. Then, protein synthesis was stopped by adding $12 \mu \mathrm{g} / \mathrm{ml}$ chloramphenicol (Sigma). Cultures were kept at $37^{\circ} \mathrm{C}$, and at different time intervals $1 \mathrm{ml}$ 
of culture was centrifuged and bacterial pellets were stored at $-20^{\circ} \mathrm{C}$. Protein samples were adjusted and analyzed by immunoblotting as described above. Western blot protein bands relative quantification was achieved using ImageJ software [17] and expressed as the ratios of the final/initial time interval signal intensities.

\subsection{Bioinformatics analysis and BPtpA structural modeling.}

Multiple protein sequence alignments were performed using MAFFT [18]. WebLogo 3.6 [19] was used to identify sequence conservation at specific positions and to compare residues between LMW-PTP classes. Bayesian inference of phylogeny was calculated using MRBAYES [20]. Structural model of BPtpA was built with the SWISS-MODEL server (http://swissmodel.expasy.org/) using $V c$ LMWPTP-1 (45.6 \% identity with BPtpA) from Vibrio cholerae O395 (PDB accession number 4LRQ) as the template. Model refinement was performed using Chiron [21]. Model visualization, and figure preparation were performed with PyMol, Version 2.0 (Schrödinger, LLC).

\section{Results}

\subsection{BPtpA belongs to the LMW-PTP class II.I.}

To clarify the functional differences between the three endogenous LMW-PTP of $B$. cenocepacia, BPtpA, BceD, and BCAM0208 [14], the amino acid sequences of these proteins were aligned with prototypical Cys-based PTP class II enzymes using Fast Fourier Transform. Based on the iterative sequence alignment, two clusters of LMW-PTP became evident (Fig. 1). The first cluster consists mainly of LMW-PTP from eukaryotes and Firmicutes, while the second contains only prokaryotic enzymes (mostly from Gramnegative bacteria), including B. cenocepacia BceD and BCAM0208 (Fig. 1). Categorization 
of the LMW-PTP into two separated clusters was further supported by its evolutionary divergence, as revealed by Bayesian inference of phylogeny (Fig. S1).

Based on structural data, Lescop et al [9] have suggested a similar distinction; they classified eukaryotic and bacterial LMW-PTP as class I and class II, respectively. Considering that LMW-PTP belong to the Cys-based PTP class II, and in keeping with the Cys-based PTP class I categorization [22] we designated the two groups found in our analyses as class II subdivision I (class II.I) and subdivision II (class II.II). The class II.I groups proteins found in eukaryotes and the majority of the LMW-PTP from Gram-positive bacteria. However, a few Gram-negative bacterial proteins, including B. cenocepacia, BPtpA also belong to class II.I (Fig. 1 and Fig. S1). The LMW-PTP class II.I are distinguished primarily by (i) a conserved aromatic residue at the W-loop commonly followed by the positively charged residue histidine, plus (ii) two consecutive aromatic residues at the D-loop (Fig. 1).

\subsection{Conserved amino acids in class II.I enzymes are important for BptpA functionality.}

To better understand the distinctive features of the two LMW-PTP groups, we examined the conserved residues within the class II.I not shared by class II.II proteins (Fig. 1). From this analysis, eight amino acid residues in BPtpA were selected for alanine replacement using site-directed mutagenesis: T44, W47, and H48 (at W-loop), D80 and F81 (at $\alpha 3$ helix), F130 (at D-loop), and D141 and C147 (at $\alpha 5$-helix). Because an equivalent W-loop aromatic residue is absent in class II.II enzymes, we also constructed a version of BPtpA lacking W47 (Table S1). 
We have previously shown that an enzymatically active C-terminal FLAG-tagged BPtpA version can restore the growth defect of B. cenocepacia $\triangle b p t p A$ strain under limiting nutrient conditions [14], indicating that the FLAG tag does not alter protein function. Therefore, plasmid pL2200 (Table S1), encoding the bptp $A_{\mathrm{FLAG}}$ chimera was used as template for site-directed mutagenesis. Each plasmid derivative (Table S1) was mobilized into B. cenocepacia $\triangle b p t p A$ strain and their growth in M9 minimal medium was assessed (Fig. 2). The results showed that BPtpA mutant versions W47A, $\Delta \mathrm{W} 47, \mathrm{H} 48 \mathrm{~A}, \mathrm{D} 80 \mathrm{~A}$, and F81A could not restore growth when expressed in $B$. cenocepacia $\Delta b p t p A$ strain, similar to BPtpA with the catalytic point mutation D127A, or the negative control plasmid pDA17 (Fig. 2A). In contrast, alanine replacements in BptpA residues T44, F130, D141, or C147 did not alter protein functionality based on the ability of these constructs to restore the $\triangle b p t p A$ strain growth at comparable levels as the parental protein (Fig. 2B). Expression of all BPtpA replacement mutants was validated by immunoblotting using a monoclonal antibody against the FLAG-tag (Fig. 2C, upper panel). Detection of recombinant BPtpA versions was not uniform. Indeed, BPtpA with D80A and F81 A replacements did not restore growth of $\triangle b p t p A$ strain, and also had reduced protein expression as compared to the wild-type enzyme (Fig. 2C, upper panel). This reduced signal was not due to differences in protein loading, as validated by detection of an unrelated cross-reacting protein by using a rabbit anti-E. coli serum (Fig. 2C, lower panel).

\subsection{Phosphatase activity and stability of BPtpA loss-of-function point mutants.}

BPtpA with substitutions W47A, $\Delta \mathrm{W} 47, \mathrm{H} 48 \mathrm{~A}, \mathrm{D} 80 \mathrm{~A}$, and F81A resemble the phenotype of the catalytic mutation D127A (Fig. 2A). Therefore, to rule out an enzymatic deficiency of this BPtpA protein versions, each corresponding gene was subcloned into an inducible 
vector (Table S1). Resulting recombinant proteins were purified by nickel affinity chromatography (Fig. 3A). Similar amount of recombinant protein was recovered from the different BPtpA versions, except for point mutants D80A and F81A. The purified protein yield (mg/l of culture) obtained for each BPtpA version was as follows: wild-type 0.91 $\pm 0.07 ;$ W47A $0.87 \pm 0.06 ; \Delta \mathrm{W} 471.03 \pm 0.21 ; \mathrm{H} 48 \mathrm{~A} 1.12 \pm 0.13 ; \mathrm{D} 80 \mathrm{~A} 0.39 \pm 0.05 ; \mathrm{F} 81 \mathrm{~A}$ $0.28 \pm 0.08 ;$ and D127A $1.30 \pm 0.31$.

Phosphatase activity of purified BPtpA point mutants was assessed using $p$-nitrophenol phosphate (Fig. 3B). The specific activity of BPtpA versions W47A, $\Delta$ W47, and H48A showed no significant differences when compared to wild-type enzyme $(1.37 \pm 0.19 \mu$ moles $\mathrm{min}^{-1} \mathrm{mg}^{-1}$ ). In contrast, the enzymatic activity of BPtpA D80A and F81 A was abrogated, similarly to the BPtpA inactive version, D127A (Fig. 3B).

Based on the results obtained for BPtpA versions D80A and F81A, we speculated that protein stability or misfolding might be responsible for the loss of function of these two mutant proteins. To confirm this, we compared the stability of BPtpA wild-type, and inactive point mutants D80A, F81A, and D127A after protein synthesis arrest (Fig. 3C). Time-course analysis of BPtpA D80A and F81A revealed a continuous decrease over the evaluated time period. In contrast, BPtpA wild-type or inactive point mutant D127A showed a constant signal during the length of the experiment (Fig. 3C). The relative densitometric quantification of the final/initial time interval intensities also evidenced the temporal detriment of BPtpA D80A and F81A, compared to wild-type or D127A (Fig. 3C).

\section{Discussion}


LMW-PTP are ubiquitous enzymes belonging to the Cys-based PTP class II family. They share high amino acid sequence identity plus a common fold [4]. Based on our results, we propose to sub-divide the LMW-PTP enzymes into two separated groups, referred as class II.I and class II.II. Using BPtpA from B. cenocepacia, as a representative member of the LMW-PTP class II.I, we demonstrate a key biological role of residues W47, H48, D80, and F81. Our BPtpA structural model (Fig. 4A and B) suggests that W-loop residues W47 and H48 are part of an exposed wall regulating the selective access of phosphorylated substrates to the inward phosphatase active site. Indeed, the structural orientation and charge topology of the W-loop in eukaryotic LMW-PTPs were implicated in the recognition of specific ligands [8]. For instance, the corresponding residue to BPtpA H48 imparts charge to the active site and isoenzyme kinetic specificity in the HPTP-A and HPTP-B human isoforms $[23,24]$. Further, P-loop ligand binding triggers a large reorientation of the residue W48 in MptpA from M. tuberculosis (W47 in BPtpA) and the D-loop, provoking the transition from an open to a closed conformation that modulates the surface of the substrate binding pocket [25].

A triad of conserved aromatic residues at W- and D-loop is the hallmark of LMW-PTP class II.I. Hence, a common mechanism involving these three residues during proper ligand positioning might be anticipated. Accordingly, strong $\pi-\pi$ stacking interactions implicating W- and D-loop aromatic residues of HPTP-A were identified as critical for binding the benzothiazole ring of different pTyr mimetic inhibitors [26]. Nonetheless, alanine replacement of the second D-loop aromatic residue (F130) did not affect either BPtpA capability to restore $\triangle b p t p A$ strain growth defect (Fig. 2B) or phosphatase activity (data not shown), indicating that at least for BPtpA, the first D-loop aromatic residue (Y129) must 
provide the main site for substrate binding. In agreement, alanine substitution of the corresponding residue in Ltp1 from Saccharomyces cerevisiae caused a marked drop in the $k_{\text {cat }} / K_{\mathrm{m}}$ value [27]. Moreover, the first D-loop aromatic residue is conserved in LMW-PTP class II.II and it has been implicated in substrate binding [28,29]. The D-loop aromatic residues could also play a role in protein dimerization, as suggested for the bovine LMWPTP, BPTP [30], although an alternative mode of dimerization was reported for $V c$ LMWPTP-1 from $V$. cholerae [31]. Therefore, the contribution of D-loop residues to oligomerization still needs clarification.

Our data reveal a critical role of two BPtpA $\alpha 3$-helix conserved residues, D80 and F81. According to our BptpA model (Fig. 4A and C), both residues are located at the interior of the protein. In particular, the benzyl side chain of F81 is buried into a hydrophobic core encompassing hydrophobic residues V76 and L98 (Fig. 4C), which are highly conserved in all LMW-PTP. Moreover, the BPtpA model suggests that D80 forms a salt bridge with the charged residue R74 (Fig. 4C). An equivalent interaction is present in different LMW-PTP class II.I structures, including human, bovine, mouse, etc. (data not shown). Together, our observations suggest that the $\alpha 3$-helix conserved residues are necessary for LMW-PTP class II.I protein stability. The uncovered functional relevance of the $\alpha 3$-helix region could provide an alternative option for rational drug design against LMW-PTP, especially considering the difficulties encountered for LMW-PTP selective drug targeting involving the charged nature and high degree of homology of PTP catalytic sites [32]. Efforts are under way to systematically identify protein substrates of B. cenocepacia phosphorylated proteins, including BPtpA substrates important for adaptation to nutrient starvation.

\section{Declaration of competing interest}


All authors declare that they have no conflicts of interest

\section{Acknowledgements}

We acknowledge M.S. Maria F. Flores, M.S. José F. Espinosa, and Dra. Norma Espinosa Sánchez for their expert technical assistance.

\section{Funding}

This study was supported by a grant from SEP-PRODEP [511-6/17-7538] to A.A.

\section{References}

[1] E. Bechet, S. Guiral, S. Torres, I. Mijakovic, A.J. Cozzone, C. Grangeasse, Tyrosinekinases in bacteria: from a matter of controversy to the status of key regulatory enzymes, Amino Acids 37 (2009) 499-507.

[2] I. Mijakovic, J. Deutscher, Protein-tyrosine phosphorylation in Bacillus subtilis: a 10year retrospective, Front Microbiol 6 (2015) 18.

[3] C. Grangeasse, S. Nessler, I. Mijakovic, Bacterial tyrosine kinases: evolution, biological function and structural insights, Philos Trans R Soc Lond B Biol Sci 367 (2012) 2640-2655. [4] A. Caselli, P. Paoli, A. Santi, C. Mugnaioni, A. Toti, G. Camici, P. Cirri, Low molecular weight protein tyrosine phosphatase: Multifaceted functions of an evolutionarily conserved enzyme, Biochim Biophys Acta 1864 (2016) 1339-1355.

[5] L. Aravind, E.V. Koonin, Phosphoesterase domains associated with DNA polymerases of diverse origins, Nucleic Acids Res 26 (1998) 3746-3752.

[6] A.J. Cozzone, C. Grangeasse, P. Doublet, B. Duclos, Protein phosphorylation on tyrosine in bacteria, Arch Microbiol 181 (2004) 171-181. 
[7] K. Kolmodin, J. Åqvist, The catalytic mechanism of protein tyrosine phosphatases revisited, FEBS Lett 498 (2001) 208-213.

[8] R. Maccari, R. Ottana, Low molecular weight phosphotyrosine protein phosphatases as emerging targets for the design of novel therapeutic agents, J Med Chem 55 2-22.

[9] E. Lescop, Y. Hu, H. Xu, W. Hu, J. Chen, B. Xia, C. Jin, The solution structure of Escherichia coli Wzb reveals a novel substrate recognition mechanism of prokaryotic low molecular weight protein-tyrosine phosphatases, J Biol Chem 281 (2006) 19570-19577.

[10] P. Drevinek, E. Mahenthiralingam, Burkholderia cenocepacia in cystic fibrosis: epidemiology and molecular mechanisms of virulence, Clin Microbiol Infect 16 (2010) 821830.

[11] V.C. Scoffone, L.R. Chiarelli, G. Trespidi, M. Mentasti, G. Riccardi, S. Buroni, Burkholderia cenocepacia Infections in Cystic Fibrosis Patients: Drug Resistance and Therapeutic Approaches, Front Microbiol 8 (2017) 1592.

[12] N.P. Mira, A. Madeira, A.S. Moreira, C.P. Coutinho, I. Sá-Correia, Genomic expression analysis reveals strategies of Burkholderia cenocepacia to adapt to cystic fibrosis patients' airways and antimicrobial therapy, PLoS One 6 (2011) e28831.

[13] A. Andrade, M.A. Valvano, A Burkholderia cenocepacia gene encoding a nonfunctional tyrosine phosphatase is required for the delayed maturation of the bacteriacontaining vacuoles in macrophages, Microbiology 160 (2014) 1332-1345.

[14] A. Andrade, F. Tavares-Carreon, M. Khodai-Kalaki, M.A. Valvano, Tyrosine Phosphorylation and Dephosphorylation in Burkholderia cenocepacia Affect Biofilm Formation, Growth under Nutritional Deprivation, and Pathogenicity, Appl Environ Microbiol 82 (2016) 843-856. 
[15] A.S. Ferreira, J.H. Leitão, S.A. Sousa, A.M. Cosme, I. Sá-Correia, L.M. Moreira, Functional analysis of Burkholderia cepacia genes bceD and bceF, encoding a phosphotyrosine phosphatase and a tyrosine autokinase, respectively: role in exopolysaccharide biosynthesis and biofilm formation, Appl Environ Microbiol 73 (2007) $524-534$.

[16] M. Romo-Castillo, A. Andrade, N. Espinosa, J. Monjarás Feria, E. Soto, M. DíazGuerrero, B. Gonzaléz-Pedrajo, EscO, a Functional and Structural Analog of the Flagellar FliJ Protein, Is a Positive Regulator of EscN ATPase Activity of the Enteropathogenic Escherichia coli Injectisome, J Bacteriol 196 (2014) 2227-2241.

[17] C.A. Schneider, W.S. Rasband, K.W. Eliceiri, NIH Image to ImageJ: 25 years of image analysis, Nat Methods 9 (2012) 671-675.

[18] K. Katoh, J. Rozewicki, K.D. Yamada, MAFFT online service: multiple sequence alignment, interactive sequence choice and visualization, Brief Bioinform (2017).

[19] G.E. Crooks, G. Hon, J.M. Chandonia, S.E. Brenner, WebLogo: a sequence logo generator, Genome Res 14 (2004) 1188-1190.

[20] J.P. Huelsenbeck, F. Ronquist, MRBAYES: Bayesian inference of phylogenetic trees, Bioinformatics 17 (2001) 754-755.

[21] S. Ramachandran, P. Kota, F. Ding, N.V. Dokholyan, Automated minimization of steric clashes in protein structures, Proteins 79 (2011) 261-270.

[22] A. Alonso, R. Pulido, The extended human PTPome: a growing tyrosine phosphatase family, FEBS J 283 (2016) 1404-1429.

[23] P. Cirri, T. Fiaschi, P. Chiarugi, G. Camici, G. Manao, G. Raugei, G. Ramponi, The molecular basis of the differing kinetic behavior of the two low molecular mass phosphotyrosine protein phosphatase isoforms, J Biol Chem 271 (1996) 2604-2607. 
[24] A.P. Zabell, A.D. Schroff, Jr., B.E. Bain, R.L. Van Etten, O. Wiest, C.V. Stauffacher, Crystal structure of the human B-form low molecular weight phosphotyrosyl phosphatase at 1.6-Å resolution, J Biol Chem 281 (2006) 6520-6527.

[25] T. Stehle, S. Sreeramulu, F. Löhr, C. Richter, K. Saxena, H.R. Jonker, H. Schwalbe, The apo-structure of the low molecular weight protein-tyrosine phosphatase A (MptpA) from Mycobacterium tuberculosis allows for better target-specific drug development, J Biol Chem 287 (2012) 34569-34582.

[26] R. He, J. Wang, Z.H. Yu, R.Y. Zhang, S. Liu, L. Wu, Z.Y. Zhang, Inhibition of Low Molecular Weight Protein Tyrosine Phosphatase by an Induced-Fit Mechanism, J Med Chem 59 (2016) 9094-9106.

[27] P. Paoli, A. Modesti, F. Magherini, T. Gamberi, A. Caselli, G. Manao, G. Raugei, G. Camici, G. Ramponi, Site-directed mutagenesis of two aromatic residues lining the active site pocket of the yeast Ltp1, Biochim Biophys Acta 1770 (2007) 753-762.

[28] G. Hagelueken, H. Huang, I.L. Mainprize, C. Whitfield, J.H. Naismith, Crystal structures of Wzb of Escherichia coli and CpsB of Streptococcus pneumoniae, representatives of two families of tyrosine phosphatases that regulate capsule assembly, $\mathrm{J}$ Mol Biol 392 (2009) 678-688.

[29] D.B. Temel, K. Dutta, S. Alphonse, J. Nourikyan, C. Grangeasse, R. Ghose, Regulatory interactions between a bacterial tyrosine kinase and its cognate phosphatase, J Biol Chem 288 (2013) 15212-15228.

[30] L. Tabernero, B.N. Evans, P.A. Tishmack, R.L. Van Etten, C.V. Stauffacher, The structure of the bovine protein tyrosine phosphatase dimer reveals a potential self-regulation mechanism, Biochemistry 38 (1999) 11651-11658. 
[31] S. Nath, R. Banerjee, U. Sen, Atomic resolution crystal structure of VcLMWPTP-1 from Vibrio cholerae 0395: insights into a novel mode of dimerization in the low molecular weight protein tyrosine phosphatase family, Biochem Biophys Res Commun 450 (2014) 390395.

[32] Z.Y. Zhang, Drugging the Undruggable: Therapeutic Potential of Targeting Protein Tyrosine Phosphatases, Acc Chem Res 50 (2017) 122-129. 
Figure 1. ( 2 column fitting image)

A

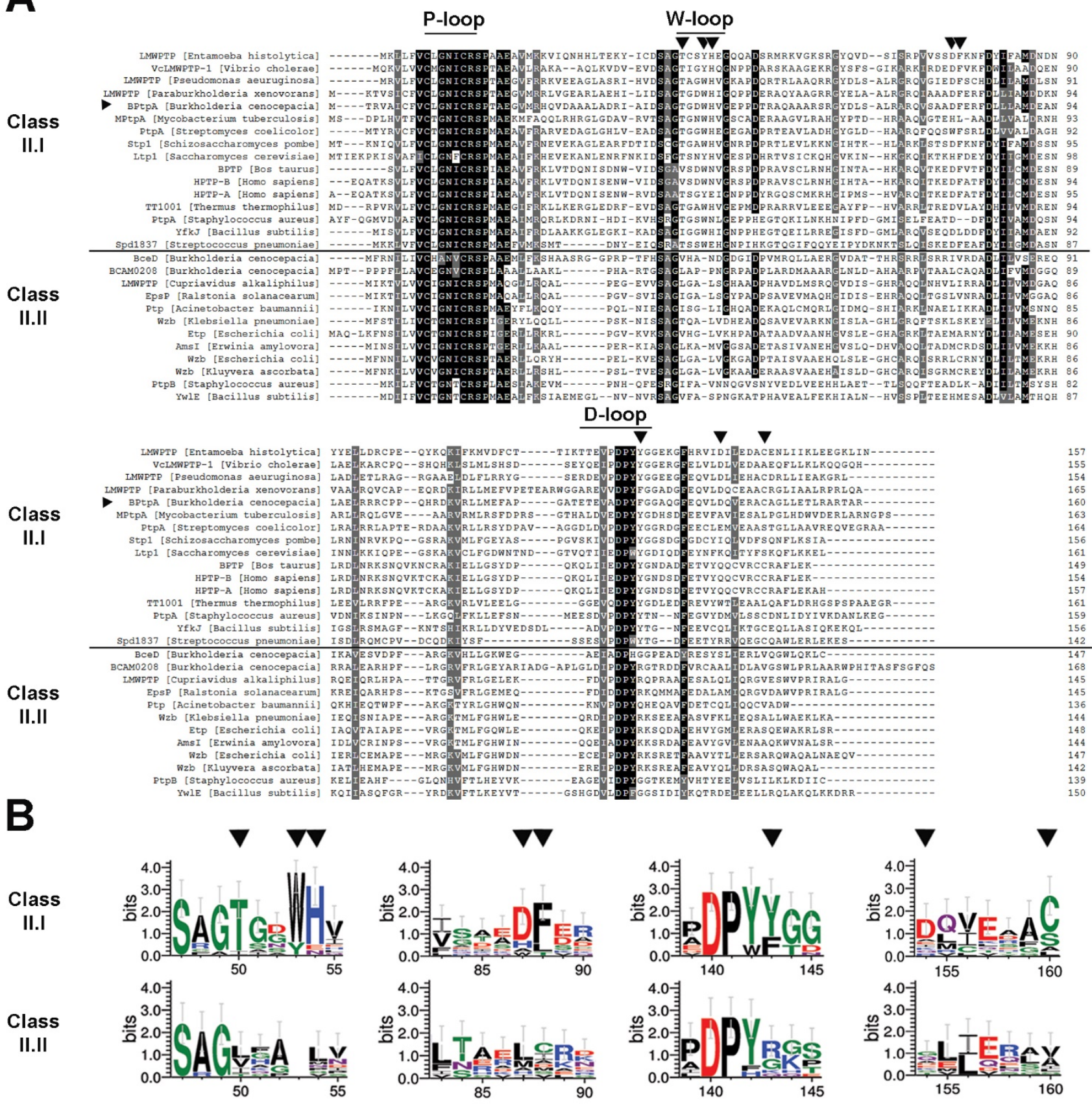

Figure 1. LMW-PTP enzymes can be categorized in two groups. Multiple sequence alignment of representative LMW-PTP enzymes. Straight horizontal line separates enzymes from Class II.I (above) and Class II.II (below). Residues composing the P-, W-, and Dloops are indicated. (B) Sequence logos for the corresponding regions in LMW-PTP Class II.I and Class II.II. Arrowheads denote BPtpA sequence, as well as the corresponding residues mutagenized in this study. 
Figure 2. (1 column fitting image)

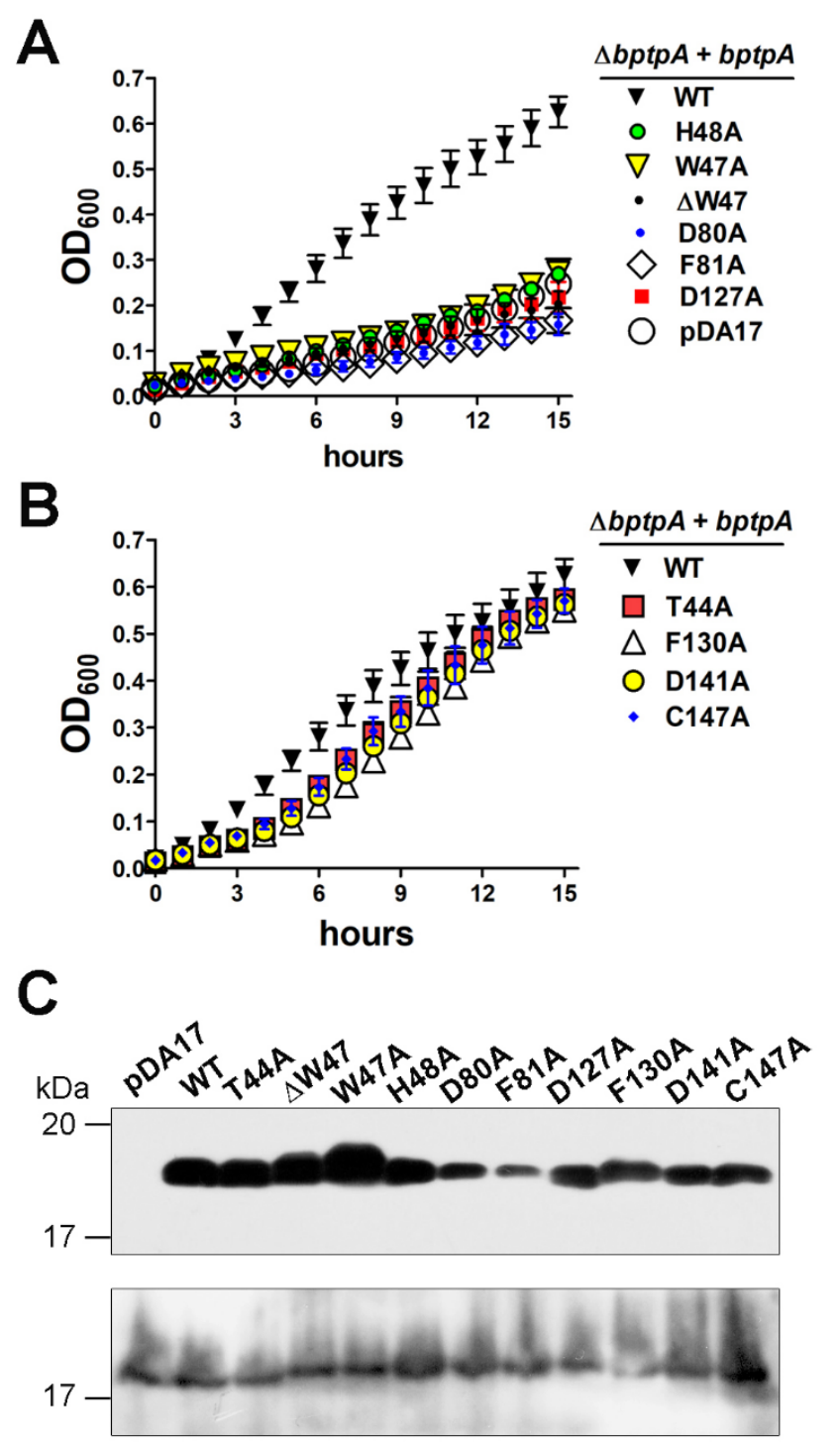

Figure 2. Conserved residues from LMW-PTP class II.I are required for BPtpA functionality. (A and B) Complementation of growth in M9 minimal medium of $B$. cenocepacia $\triangle b p t p A$ carrying $\mathrm{pDA} 17$-based plasmids. Data represent the mean $\pm \mathrm{SEM}$ from three independent experiments. (C) Immunoblot of total cell lysates of B. cenocepacia $\triangle b p t p A$ carrying pDA17-based plasmids growing in M9 minimal medium. Upper and lower panels, immunoblot with anti-FLAG antibody or anti-E. coli serum, respectively. The positions of molecular mass markers are indicated on the left. Immunoblots were repeated at least three times. 
Figure 3. (1.5 column fitting image)

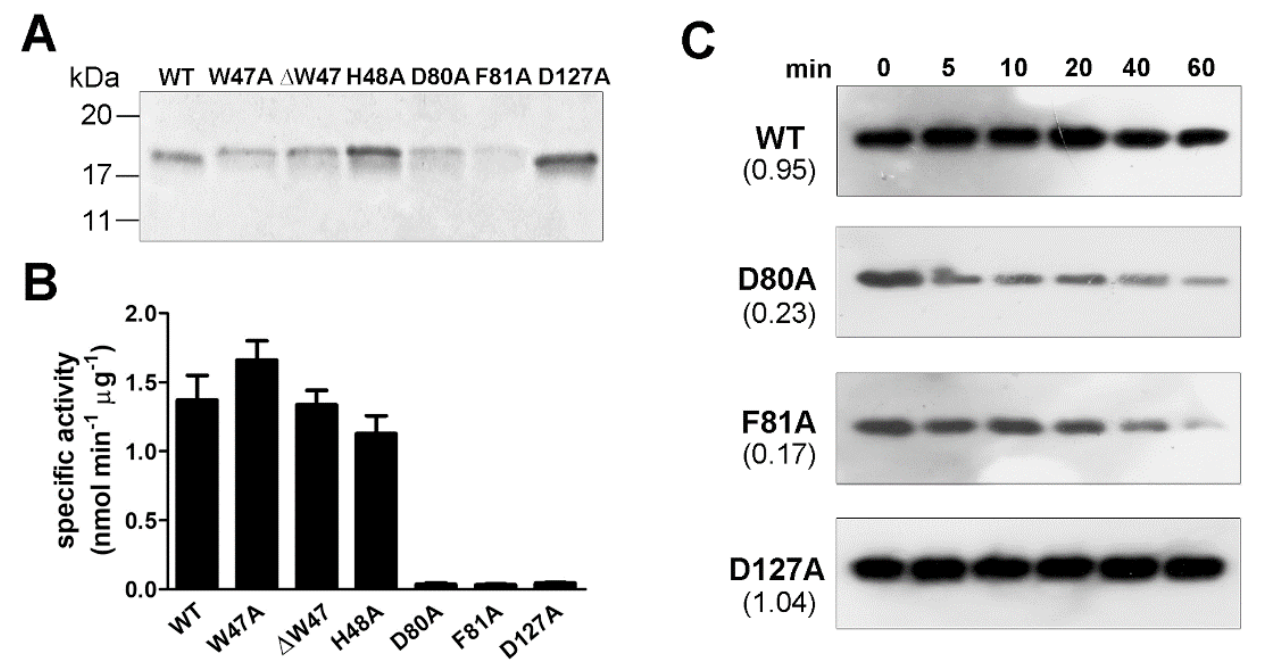

Figure 3. Phosphatase activity and stability of loss-of-function BPtpA point mutants. (A) Purification of recombinant LMW-PTP, each line corresponds to the indicated BPtpA version. The positions of molecular mass markers are indicated on the left. (B) In vitro phosphatase activity of purified BPtpA recombinant protein using $p$-nitrophenol phosphate (PNPP) as substrate. Data represent the mean \pm SEM from three independent experiments. (C) In vivo protein stability of BPtpA wild-type (WT) and inactive point mutant versions after stopping protein synthesis. At the indicated time intervals (minutes), a sample was collected and frozen. Immunoblot was performed with anti-FLAG antibody. The ratio final/initial signal intensity is indicated in parenthesis under the corresponding BPtpA version. Protein stability assays were performed three times. 
A

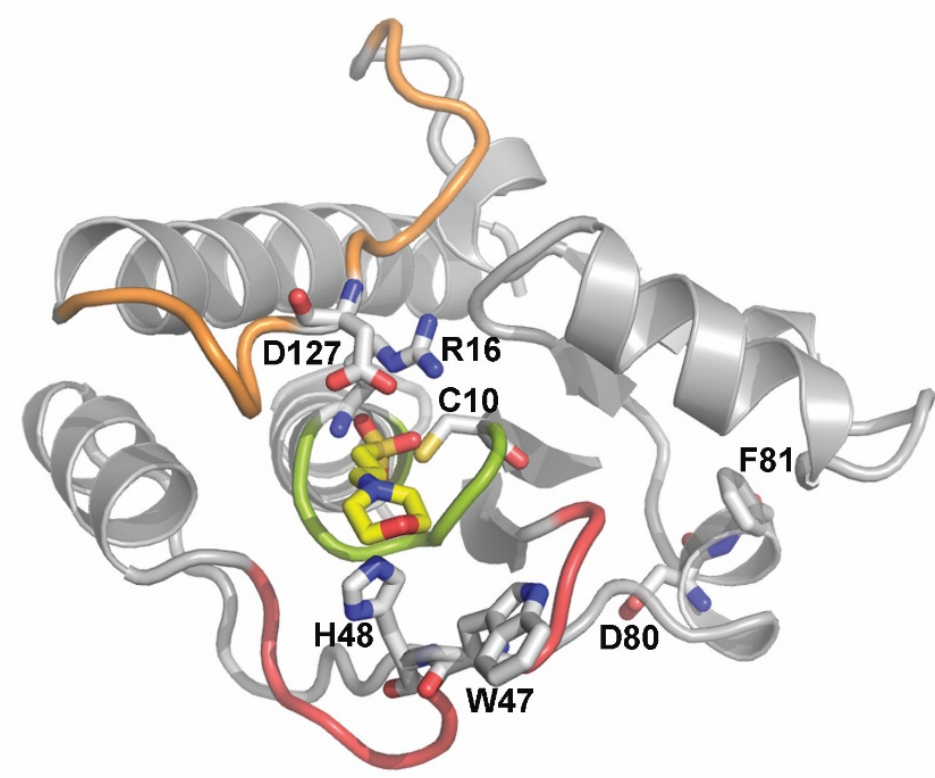

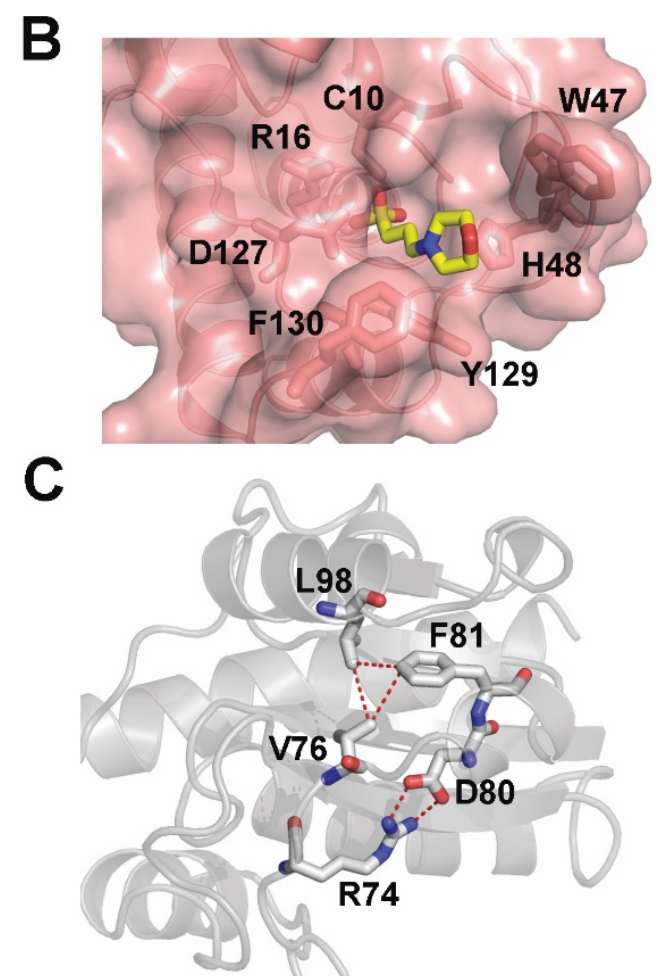

Figure 4. Mapping of functional residues on the BPtpA structural model. Modelled BPtpA is represented as a cartoon. (A) Functional residues identified in this work W47, H48, D80, and F81 are depicted as sticks. The P-, D-, and W-loop are colored in green, orange, and red, respectively. (B) Surface representation of the active-site cavity. Aromatic residues at W-loop, W47 and H48, and D-loop, Y129 and F130, are depicted as sticks. In (A and B) MOPS as ligand mimicking the substrate phosphotyrosine at the active site is shown as yellow sticks. Catalytic residues C10, R16 and D127 are depicted as sticks. (C) Close-up view of the $\alpha 3$-helix residues D80 and F81 residues and the presumptive interactions with R74, V76, and L98, respectively. 


\section{Credit Author Statement}

AA- Conceptualization, Methodology, Resources, Funding acquisition, Writing-original draft,

Writing-review \& editing, Project administration; RSR- Investigation, Methodology, Visualization;

GMG- Resources, Writing-review \& editing; MABG- Resources, Methodology, Formal Analysis;

RJTR- Investigation, Formal Analysis; Writing-review \& editing; AMV- Investigation, Validation;

BGP- Methodology, Resources, Writing-review \& editing; MV-Resources, Writing-original draft, Writing-review \& editing. 
Table S1. Strains and plasmids ${ }^{\mathrm{a}}$

\begin{tabular}{|c|c|c|}
\hline Strain or plasmid & Relevant characteristics & Source/reference \\
\hline \multicolumn{3}{|l|}{ B. cenocepacia strains } \\
\hline K56-2 & ET12 clone related to $\mathrm{J} 2315$, clinical isolate & [1] \\
\hline$\triangle B C A L 2200(\triangle b p t p A)$ & K56-2 derivative with $\triangle B C A L 2200(\triangle b p t p A)$ & [2] \\
\hline \multicolumn{3}{|l|}{ E. coli strains } \\
\hline BL21 (DE3) & 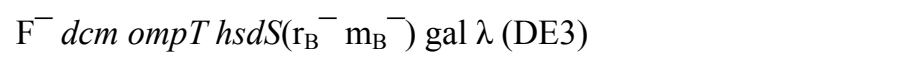 & Stratagene \\
\hline DH5 $\alpha$ & 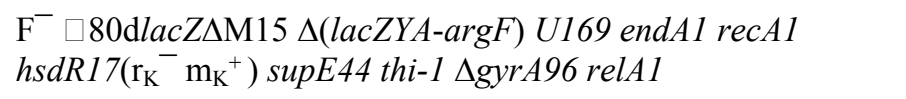 & Laboratory stock \\
\hline \multicolumn{3}{|l|}{ Plasmids } \\
\hline pDA17 & $\begin{array}{l}\text { Burkholderia-expressing vector, ori }{ }_{\mathrm{pBBR} 1} \mathrm{Tet}^{\mathrm{r}} \mathrm{mob}^{+} \mathrm{P}_{\mathrm{dhfr}} \text { FLAG } \\
\text { epitope }\end{array}$ & $\begin{array}{l}\text { D. Aubert, } \\
\text { unpublished }\end{array}$ \\
\hline pET28 & Expression vector, ori $i_{\mathrm{pBBR} 1} \operatorname{Kan}^{r} P_{T 7}$ & Novagen \\
\hline pRK2013 & ori $i_{\text {colEl } 1}$, RK2 derivative, $\mathrm{Kan}^{\mathrm{r}} \mathrm{mob}^{+} \operatorname{tra}^{+}$ & {$[3]$} \\
\hline pL2200 & pDA17 carrying B. cenocepacia BPtpA & {$[2]$} \\
\hline $\operatorname{pbptpA}_{\mathrm{T} 44 \mathrm{~A}}$ & pL2200 with the T44A substitution & This study \\
\hline $\operatorname{pbptp}_{\mathrm{W} 47 \mathrm{~A}}$ & pL2200 with the W47A substitution & This study \\
\hline $\operatorname{pbptpA}_{\Delta \mathrm{W} 47}$ & pL2200 with the $\Delta \mathrm{W} 47$ substitution & This study \\
\hline pbptpA $\mathrm{A}_{\mathrm{H} 48 \mathrm{~A}}$ & pL2200 with the H48A substitution & This study \\
\hline $\operatorname{pbptpA}_{\mathrm{D} 80 \mathrm{~A}}$ & pL2200 with the D80A substitution & This study \\
\hline pbptpA $A_{F 81 A}$ & pL2200 with the F81A substitution & This study \\
\hline $\mathrm{pL} 2200_{\mathrm{D} 127 \mathrm{~A}}$ & pL2200 with the D127A substitution & {$[4]$} \\
\hline $\operatorname{pbptpA}_{\mathrm{F} 130 \mathrm{~A}}$ & pL2200 with the F130A substitution & This study \\
\hline $\operatorname{pbptpA}_{\mathrm{D} 141 \mathrm{~A}}$ & pL2200 with the D141A substitution & This study \\
\hline $\operatorname{pbptpA}_{\mathrm{C} 147 \mathrm{~A}}$ & pL2200 with the C147A substitution & This study \\
\hline pAT44 & pET28 carrying $6 \times$ His-tagged $B$. cenocepacia bptpA & {$[4]$} \\
\hline pRS1 & pAT44 with the T44A substitution & This study \\
\hline pRS2 & pAT44 with the W47A substitution & This study \\
\hline
\end{tabular}


${ }^{\mathrm{a} K a n}$, kanamycin; Tet, tetracycline.

Table S2. Oligonucleotide primers

\begin{tabular}{|c|c|c|c|}
\hline Primer & $5^{\prime}-3^{\prime}$ primer sequence ${ }^{a}$ & $\begin{array}{l}\text { Restriction } \\
\text { enzyme }^{b}\end{array}$ & Purpose \\
\hline 001 & 5' CGGCCGGCACCGGCGACCACGTGGGCGAGCCGCC3' & NA & \multirow{2}{*}{$\begin{array}{l}\text { Pair to eliminate } \\
\text { bptpA W47 }\end{array}$} \\
\hline 002 & 5' GGCGGCTCGCCCACGTGGTCGCCGGTGCCGGCCG 3' & NA & \\
\hline 003 & 5' GTGAGCGCGGCGGATGCCGAGCGGTTCGATCTG 3' & NA & \multirow{2}{*}{$\begin{array}{l}\text { Pair to exchange } \\
\text { bptpA F81A }\end{array}$} \\
\hline 004 & 5' CAGATCGAACCGCTCGGCATCCGCCGCGCTCAC 3' & NA & \\
\hline 7506 & 5' GATCGATTCGGCCGGCGCCGGCGACTGGCACGTG 3' & NA & \multirow{2}{*}{$\begin{array}{l}\text { Pair to exchange } \\
\text { bptpA T44A }\end{array}$} \\
\hline 7507 & 5' CACGTGCCAGTCGCCGGCGCCGGCCGAATCGATC 3' & NA & \\
\hline 7508 & 5' CAGGTGAGCGCGGCGGCGTTCGAGCGGTTCGATC 3' & NA & \multirow{2}{*}{$\begin{array}{l}\text { Pair to exchange } \\
\text { bptpA D } 80 \mathrm{~A}\end{array}$} \\
\hline 7509 & 5' GATCGAACCGCTCGAACGCCGCCGCGCTCACCTG 3' & NA & \\
\hline 7512 & 5' CTTCGAACAGGTGCTCGCCCAGGTCGAGCGCGCG 3' & NA & \multirow{2}{*}{$\begin{array}{l}\text { Pair to exchange } \\
\text { bptpA D141A }\end{array}$} \\
\hline 7513 & 5' CGCGCGCTCGACCTGGGCGAGCACCTGTTCGAAG 3' & NA & \\
\hline 7514 & 5' CCAGGTCGAGCGCGCGGCCGCGGGCCTGCTGGAG3' & NA & \multirow{2}{*}{$\begin{array}{l}\text { Pair to exchange } \\
\text { bptpA C147A }\end{array}$} \\
\hline 7515 & 5' CTCCAGCAGGCCCGCGGCCGCGCGCTCGACCTGG 3' & NA & \\
\hline 7575 & 5' GCCGGCACCGGCGACGCGCACGTGGGCGAGCC 3' & NA & \multirow{2}{*}{$\begin{array}{l}\text { Pair to exchange } \\
\text { bptpA W47A }\end{array}$} \\
\hline 7576 & 5' GGCTCGCCCACGTGCGCGTCGCCGGTGCCGGC 3' & NA & \\
\hline 7577 & 5' GGCACCGGCGACTGGGCCGTGGGCGAGCCGCCC 3' & NA & \multirow{2}{*}{$\begin{array}{l}\text { Pair to exchange } \\
\text { bptpA H48A }\end{array}$} \\
\hline 7578 & 5' GGGCGGCTCGCCCACGGCCCAGTCGCCGGTGCC 3' & NA & \\
\hline
\end{tabular}




\begin{tabular}{|c|c|c|c|}
\hline 7579 & 5' GTGGCCGATCCGTATGCCGGCGGTGCGCAGGGC 3' & NA & Pair to exchange \\
\hline 7580 & 5' GCCCTGCGCACCGCCGGCATACGGATCGGCCAC 3' & NA & $b p t p A \mathrm{~F} 130 \mathrm{~A}$ \\
\hline 6008 & 5' TTTCATATGACCCGCGTTGCGATC 3' & NdeI & $\begin{array}{l}\text { Forward primer for } \\
\text { bptpA }\end{array}$ \\
\hline 6009 & 5' TTTTCTAGAGCGCGCCGTGCGGGC 3' & $X b a \mathrm{I}$ & $\begin{array}{l}\text { Reverse primer for } \\
\text { bptpA }\end{array}$ \\
\hline 7271 & 5' TTTCCATGGCCCGCGTTGCGATCTG 3' & NcoI & $\begin{array}{l}\text { Forward primer for } \\
\text { bptpA }\end{array}$ \\
\hline 7272 & 5' TTTCTCGAGGCGCGCCGTGCGGGCC 3' & XhoI & $\begin{array}{l}\text { Reverse primer for } \\
\text { bptpA }\end{array}$ \\
\hline
\end{tabular}

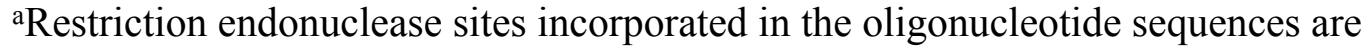
underlined.

${ }^{b} \mathrm{NA}$, absence of a restriction site.

\section{References}

[1] E. Mahenthiralingam, T. Coenye, J.W. Chung, D.P. Speert, J.R. Govan, P. Taylor, P. Vandamme, Diagnostically and experimentally useful panel of strains from the Burkholderia cepacia complex, J Clin Microbiol 38 (2000) 910-913.

[2] A. Andrade, M.A. Valvano, A Burkholderia cenocepacia gene encoding a nonfunctional tyrosine phosphatase is required for the delayed maturation of the bacteria-containing vacuoles in macrophages, Microbiology 160 (2014) 1332-1345.

[3] D.H. Figurski, D.R. Helinski, Replication of an origin-containing derivative of plasmid RK2 dependent on a plasmid function provided in trans, Proc Natl Acad Sci U S A 76 (1979) 1648-1652.

[4] A. Andrade, F. Tavares-Carreon, M. Khodai-Kalaki, M.A. Valvano, Tyrosine Phosphorylation and Dephosphorylation in Burkholderia cenocepacia Affect Biofilm Formation, Growth under Nutritional Deprivation, and Pathogenicity, Appl Environ Microbiol 82 (2016) 843-856. 
Supplementary Figure 1. Bayesian inference phylogenetic tree of representative LMWPTP. The two major clades corresponding to Class II.I and Class II.II enzymes are indicated.

YwIE_Bacillus_subtilis PtpB_Staphylococcus_aureus

Class II.II BCeD_Burkholderia_cenocepacia

-Wzb_Kluyvera_ascorbata

-Wzb_Escherichia_coli

AmsI_Enwinia_amylovora

_Etp_Escherobsiella_pneumoniae

-TT1001_Thermus_thermophilus

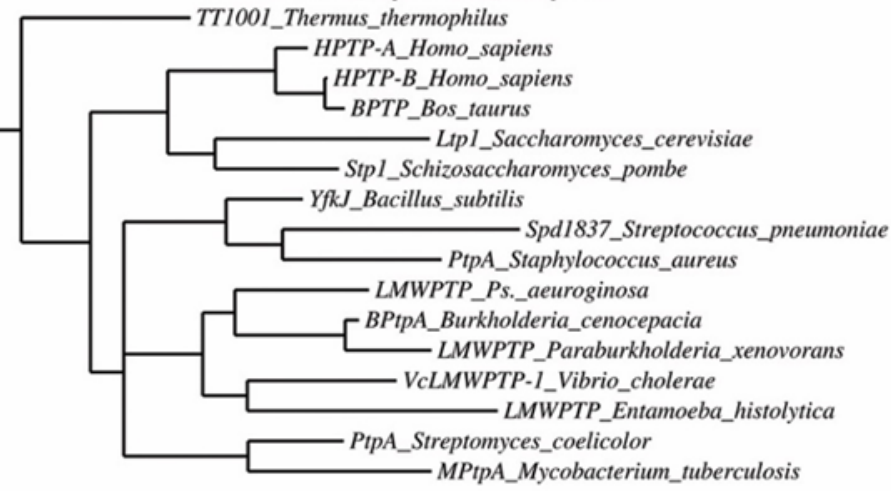

0.3 